\title{
Hopf Bifurcation Analysis of a Two-Delay HIV-1 Virus Model with Delay-Dependent Parameters
}

\author{
Yu Xiao $\mathbb{D}^{1},{ }^{1}$ Yunxian Dai $\mathbb{D}^{1},{ }^{1}$ and Jinde Cao $\mathbb{D}^{2,3}$ \\ ${ }^{1}$ Department of Applied Mathematics, Kunming University of Science and Technology, Kunming, Yunnan 650500, China \\ ${ }^{2}$ School of Mathematics, Southeast University, Nanjing, Jiangsu 210096, China \\ ${ }^{3}$ Yonsei Frontier Lab, Yonsei University, Seoul 03722, Republic of Korea \\ Correspondence should be addressed to Yunxian Dai; dyxian1976@sina.com
}

Received 17 July 2021; Accepted 11 October 2021; Published 8 November 2021

Academic Editor: Mohammad Yaghoub Abdollahzadeh Jamalabadi

Copyright (c) $2021 \mathrm{Yu}$ Xiao et al. This is an open access article distributed under the Creative Commons Attribution License, which permits unrestricted use, distribution, and reproduction in any medium, provided the original work is properly cited.

In this paper, a two-delay HIV-1 virus model with delay-dependent parameters is considered. The model includes both virusto-cell and cell-to-cell transmissions. Firstly, immune-inactivated reproduction rate $R_{0}$ and immune-activated reproduction rate $R_{1}$ are deduced. When $R_{1}>1$, the system has the unique positive equilibrium $E^{*}$. The local stability of the positive equilibrium and the existence of Hopf bifurcation are obtained by analyzing the characteristic equation at the positive equilibrium with the time delay as the bifurcation parameter and four different cases. Besides, we obtain the direction and stability of the Hopf bifurcation by using the center manifold theorem and the normal form theory. Finally, the theoretical results are validated by numerical simulation.

\section{Introduction}

AIDS is a very dangerous infectious disease caused by HIV-1 virus which attacks the human immune system. At present, it has been proved that there are two different mechanisms for the spread of HIV-1 virus in the host: virus-cell transmission and cell-cell transmission [1]. A great number of studies have considered the above two contagion mechanisms [1-5]. Wang and Zou [4] considered an HIV-I model with humoral immunity, which is a typical virus-to-cell transmissions. In 2017, Lin et al. [5] proposed a cell-to-cell transmission model and described global threshold dynamics of the model.

In recent years, delay differential equation has attracted extensive attention worldwide. It has important applications in many fields such as physics, information, economy, and biomathematics. Depending on the different circumstances, many differential equation models with single delay or multiple delays have been proposed and studied deeply [4, 6-10]. Dong et al. [6] studied the dynamics of the tumor immune system interaction model and investigated the existence of Hopf bifurcation with two time delays as bifurcation parameters. In [7], the authors discussed the influence of awareness coverage and time delays on infectious diseases and found that the endemic equilibrium existed a Hopf bifurcation in both delayed and nondelayed system. A two-delay model with Holling II functional response and stage structure is considered in [8]. The authors have investigated a predator-prey model with a class of Beddington-DeAngelis functional response and two delays in [9]. Recently, in [10], a two-delay HIV-1 virus model with virus-to-cell and cell-to-cell transmission is considered as follows: 


$$
\left\{\begin{array}{l}
\frac{\mathrm{d} x(t)}{\mathrm{d} t}=r x(t)\left(1-\frac{x(t)}{x_{M}}\right)-\beta_{1} x(t) v(t) \\
-\beta_{2} x(t) y(t) \\
\frac{\mathrm{d} y(t)}{\mathrm{d} t}=\beta_{1} \alpha x\left(t-\tau_{1}\right) v\left(t-\tau_{1}\right) \\
+\beta_{2} \alpha x\left(t-\tau_{1}\right) y\left(t-\tau_{1}\right)-a y(t) \\
\frac{\mathrm{d} v(t)}{\mathrm{d} t}=k y(t)-u v(t)-p v(t) z(t) \\
\frac{\mathrm{d} z(t)}{\mathrm{d} t}=c v\left(t-\tau_{2}\right) z\left(t-\tau_{2}\right)-b z(t)
\end{array}\right.
$$

where $x(t), y(t), v(t)$, and $z(t)$ represent the susceptible cells, infected cells, virus, and B cells, respectively. $x_{M}$ is the number of carrying target cells. $\beta_{1}$ and $\beta_{2}$ denote infection rates of virus-to-cell transmission and cell-tocell transmission. $r$ and $b$ are the growth and death rate of susceptible cells. $a$ and $u$ stand for the mortality rates of infected cells and virus, respectively. $k$ is the number of free virus particles produced by per infected cell. $\alpha$ is the surviving probability the time period from $t-\tau_{1}$ to $t$. $p v(t) z(t)$ describes the virus killed by B cells, and $c v(t) z(t)$ represents the new B cells produced when stimulated by antigen. The time delay $\tau_{1}$ stands for the time between virus entering into a cell and producing new virus or the time between infected cells spreading virus into uninfected cells and producing new virus. And $\tau_{2}$ is the time that the HIV-1 virus stimulates the production of $B$ cells.

In [10], the coefficients of equations are considered to be constants independent of the time delay. The authors investigated the stability of positive equilibrium and the existence of Hopf bifurcation. The explicit formula for determining the direction of Hopf bifurcation and the stability of bifurcating periodic solutions was derived. However, in practical situations, virus-cell transmission or cell-cell transmission is not instantaneous but takes some time defined by the average evaluation period $\tau$. Moreover, the growth of virus at $t$ is decided by the number of infected cells at $t-\tau$ and still alive at $t$. On this occasion, it is inappropriate to regard survival rate as a constant. Let $m$ be death rate of infected cells, then the survival rate is $e^{-m \tau}$ from $t-\tau$ to $t$. Adding time delays to parameters is helpful to further explore the complex dynamic behavior of delay differential equations and their generation mechanism.
Based on Sun and Li'work, considering that the two contagion processes are not instantaneous and the probability of cell survival is proportional to the number of cells that can survive to time $t$ at $t-\tau_{1}$, we propose the following delay model:

$$
\left\{\begin{array}{l}
\frac{\mathrm{d} x(t)}{\mathrm{d} t}=r x(t)\left(1-\frac{x(t)}{x_{M}}\right)-\beta_{1} x(t) v(t)-\beta_{2} x(t) y(t) \\
\quad \frac{\mathrm{d} y(t)}{\mathrm{d} t}=\beta_{1} e^{-m \tau_{1}} x\left(t-\tau_{1}\right) v\left(t-\tau_{1}\right) \\
+\beta_{2} e^{-m \tau_{1}} x\left(t-\tau_{1}\right) y\left(t-\tau_{1}\right)-a y(t) \\
\frac{\mathrm{d} v(t)}{\mathrm{d} t}=k y(t)-u v(t)-p v(t) z(t) \\
\frac{\mathrm{d} z(t)}{\mathrm{d} t}=c v\left(t-\tau_{2}\right) z\left(t-\tau_{2}\right)-b z(t)
\end{array}\right.
$$

where $e^{-m \tau_{1}}$ is the probability of surviving the time period from $t-\tau_{1}$ to $t$ in case $m$ is the death rate of infected but not yet virus-producing cells.

The initial conditions for system (2) are given as

$$
\begin{aligned}
x(\theta) & =\phi_{1}(\theta), \\
y(\theta) & =\phi_{2}(\theta), \\
v(\theta) & =\phi_{3}(\theta), \\
z(\theta) & =\phi_{4}(\theta), \\
\phi_{i}(\theta) & \geq 0, \\
\theta & \in[-\tau, 0), \\
\phi_{i}(0) & >0, \\
i & =1,2,3,4 .
\end{aligned}
$$

A number of scholars investigate the delay differential equations with delay-dependent parameters [11, 12]. Song et al. [11] studied a delayed viral infection model with lytic immune response. The characteristic equations of the system are like $P_{0}(\lambda, \tau)+P_{1}(\lambda, \tau) e^{-\lambda \tau}=0$. They discussed the influence of delay on the stability of the equilibrium and the local and global asymptotic stability of the disease-free equilibrium. Li and $\mathrm{Ma}$ introduced a method for determining the stability of the characteristic equations $P_{0}(\lambda, \tau)+$ $P_{1}(\lambda, \tau) e^{-\lambda \tau}+P_{2}(\lambda, \tau) e^{-2 \lambda \tau}=0$ with delay-dependent parameters in [13]. Reference [14] presented a practical geometric method to study the stability switching properties of the characteristic equation $P_{0}\left(\lambda, \tau_{1}\right)+P_{1}\left(\lambda, \tau_{1}\right) e^{-\lambda \tau_{1}}+P_{2}\left(\lambda, \tau_{1}\right) e^{-\lambda\left(\tau_{1}+\tau_{2}\right)}=0 \quad$ which 
may result from a stability analysis of a model with two time delays and delay-dependent parameters that depend only on one of the time delay. Jiang and Guo [15] studied a model with double delays and a delay-dependent parameter considering the interaction between nutrients and plankton. The authors took the time delay as the parameter to carry out the dynamic analysis of the system, including the equilibrium stability and Hopf bifurcation existence by using the method in [14].

This paper's goal is to delve into the local stability and Hopf bifurcation analysis of a two-delay HIV-1 virus model with delay-dependent parameters. The luminescent spots are as follows: (1) in this paper, two different spreads of HIV-1 virus are studied, and we will introduce time delay into the coefficients to be more realistic; (2) the conditions of the local stability of positive equilibrium and the existence of Hopf bifurcation are discussed with the time delay as the bifurcation parameters; and (3) the characteristic equation is $P_{0}(\lambda, \tau)+P_{1}(\lambda, \tau) e^{-\lambda \tau}+P_{2}(\lambda, \tau) e^{-2 \lambda \tau}=0 \quad$ when $\tau_{1}=\tau_{2}=\tau$, and the stability is determined by the geometric stability switch criterion $[13,14]$; and (4) the influences of different delay values on system stability are investigated.

The rest of this paper is organized as follows. In Section 2 , the existence of equilibrium is given. By analyzing the characteristic equation, we discuss the stability of the positive equilibrium and existence of Hopf bifurcation in four different cases. In Section 3, we consider $\tau_{2}$ as a parameter and determine the direction and stability of Hopf bifurcation by using the center manifold theorem and the normal form theory. In Section 4, we perform numerical simulations to illustrate our results. Finally, the conclusions of the paper are given in Section 5.

\section{Stability of the Positive Equilibrium and Existence of Hopf Bifurcation}

Similar to [10], we can derive immune-inactivated reproduction rate $R_{0}=\left(\left(k \beta_{1}+u \beta_{2}\right) / a u\right) x_{M} e^{-m \tau_{1}}$ and immune- activated reproduction rate $R_{1}=\operatorname{ckr} x_{M}\left(k \beta_{1}+u \beta_{2}\right) /\left(x_{M}\right.$ $\left.\left(k \beta_{1}+u \beta_{2}\right)\left(k b \beta_{1}+u b \beta_{2}\right)+c k r a u e^{m \tau_{1}}\right)=R_{0} /\left(1+b\left(k \beta_{1}\right.\right.$ $\left.+u \beta_{2}\right) R_{0} / c k r$ ), and then the equilibria of system (2) are as follows:

(i) $R_{1}<R_{0}<1$, then system (2) only has an infectionfree equilibrium $E_{0}=\left(x_{M}, 0,0,0\right)$.

(ii) $R_{1}<1<R_{0}$, then system (2) has a no-immune response equilibrium $E_{1}=\left(x_{1}, y_{1}, v_{1}, 0\right)$ except for $E_{0}$, where

$$
\begin{aligned}
& x_{1}=\frac{a u e^{m \tau_{1}}}{k \beta_{1}+u \beta_{2}}, \\
& y_{1}=\frac{u r}{k \beta_{1}+u \beta_{2}}\left(1-\frac{1}{R_{0}}\right), \\
& v_{1}=\frac{k r}{k \beta_{1}+u \beta_{2}}\left(1-\frac{1}{R_{0}}\right) .
\end{aligned}
$$

(iii) $1<R_{1}<R_{0}$, then system (2) has an immunity-activated equilibrium $E^{*}=\left(x^{*}, y^{*}, v^{*}, z^{*}\right)$ except for $E_{0}$ and $E_{1}$, where

$$
\begin{aligned}
& x^{*}=\frac{a\left(u b+p b z^{*}\right) e^{m \tau_{1}}}{k b \beta_{1}+\beta_{2}\left(u b+p b z^{*}\right)}, \\
& y^{*}=\frac{u b+p b z^{*}}{c k}, \\
& v^{*}=\frac{b}{c}
\end{aligned}
$$

and $z^{*}$ is a positive, real root of the following quadratic equation:

$$
\begin{gathered}
p^{2} \beta_{2}^{2} b x_{M} z(t)^{2}+\left[a c k r p e^{m \tau_{1}}+\beta_{2} p x_{M}\left(-c k r+2 k b \beta_{1}+2 u b \beta_{2}\right)\right] z(t) \\
+\left[x_{M}\left(k \beta_{1}+u \beta_{2}\right)\left(k b \beta_{1}+u b \beta_{2}\right)+c k r a u e^{m \tau_{1}}\right]\left(1-R_{1}\right)=0 .
\end{gathered}
$$


The characteristic equation of system (2) at $E(x, y, v, z) \quad$ where is

$$
\operatorname{det}\left(\lambda I-A-B e^{-\lambda \tau_{1}}-C e^{-\lambda \tau_{2}}\right)=0
$$

$$
\begin{aligned}
& A=\left(\begin{array}{cccc}
r-\frac{2 r x}{x_{M}}-\beta_{1} v-\beta_{2} y & -\beta_{2} x & -\beta_{1} x & 0 \\
0 & -a & 0 & 0 \\
0 & k & -u-p z & -p v \\
0 & 0 & 0 & -b
\end{array}\right) \triangleq\left(\begin{array}{cccc}
a_{11} & a_{12} & a_{13} & 0 \\
0 & a_{22} & 0 & 0 \\
0 & a_{32} & a_{33} & a_{34} \\
0 & 0 & 0 & a_{44}
\end{array}\right) \text {, }
\end{aligned}
$$

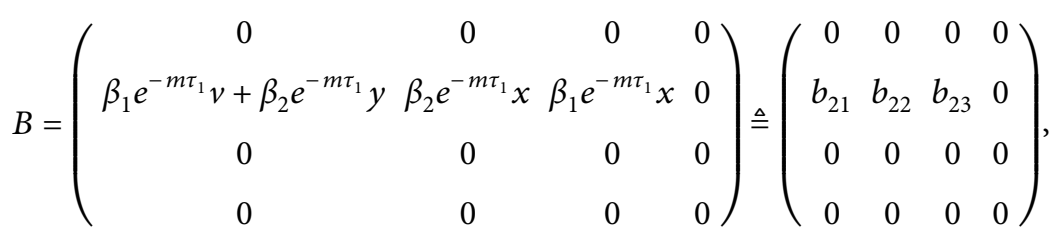

$$
\begin{aligned}
& C=\left(\begin{array}{cccc}
0 & 0 & 0 & 0 \\
0 & 0 & 0 & 0 \\
0 & 0 & 0 & 0 \\
0 & 0 & c z & c v
\end{array}\right) \triangleq\left(\begin{array}{cccc}
0 & 0 & 0 & 0 \\
0 & 0 & 0 & 0 \\
0 & 0 & 0 & 0 \\
0 & 0 & c_{43} & c_{44}
\end{array}\right)
\end{aligned}
$$

that is,

$$
\begin{aligned}
& \lambda^{4}+A_{1} \lambda^{3}+A_{2} \lambda^{2}+A_{3} \lambda+A_{4}+\left(B_{1} \lambda^{3}+B_{2} \lambda^{2}+B_{3} \lambda+B_{4}\right) e^{-\lambda \tau_{1}}+\left(C_{1} \lambda^{3}+C_{2} \lambda^{2}+C_{3} \lambda+C_{4}\right) e^{-\lambda \tau_{2}} \\
& \quad+\left(D_{1} \lambda^{2}+D_{2} \lambda+D_{3}\right) e^{-\lambda\left(\tau_{1}+\tau_{2}\right)}=0
\end{aligned}
$$

where

$$
\begin{aligned}
& A_{1}=-\left(a_{11}+a_{22}+a_{33}+a_{44}\right) \\
& A_{2}=a_{11} a_{22}+a_{11} a_{33}+a_{11} a_{44}+a_{22} a_{33}+a_{22} a_{44}+a_{33} a_{44}, \\
& A_{3}=-\left(a_{11} a_{22} a_{33}+a_{11} a_{22} a_{44}+a_{11} a_{33} a_{44}+a_{22} a_{33} a_{44}\right) \\
& A_{4}=a_{11} a_{22} a_{33} a_{44} \\
& B_{1}=-b_{22} \\
& B_{2}=a_{11} b_{22}-a_{12} b_{21}-a_{32} b_{23}+a_{33} b_{22}+a_{44} b_{22}, \\
& B_{3}=a_{11} a_{32} b_{23}-a_{11} a_{33} b_{22}+a_{12} a_{33} b_{21}-a_{13} a_{32} b_{21}-a_{11} a_{44} b_{22}+a_{12} a_{44} b_{21}+a_{32} a_{44} b_{23}-a_{33} a_{44} b_{22}, \\
& B_{4}=-a_{11} a_{32} a_{44} b_{23}+a_{11} a_{33} a_{44} b_{22}-a_{12} a_{33} a_{44} b_{21}+a_{13} a_{32} a_{44} b_{21}, \\
& C_{1}=-c_{44}, C_{2}=a_{11} c_{44}+a_{22} c_{44}+a_{33} c_{44}-a_{34} c_{43}, \\
& C_{3}=-a_{11} a_{22} c_{44}-a_{11} a_{33} c_{44}+a_{11} a_{34} c_{43}-a_{22} a_{33} c_{44}+a_{22} a_{34} c_{43}, \\
& C_{4}=a_{11} a_{22} a_{33} c_{44}-a_{11} a_{22} a_{34} c_{43}, D_{1}=b_{22} c_{44}, \\
& D_{2}=-a_{11} b_{22} c_{44}+a_{12} b_{21} c_{44}+a_{32} b_{23} c_{44}-a_{33} b_{22} c_{44}+a_{34} b_{22} c_{43}, \\
& D_{3}=-a_{11} a_{32} b_{23} c_{44}+a_{11} a_{33} b_{22} c_{44}-a_{11} a_{34} b_{22} c_{43}-a_{12} a_{33} b_{21} c_{44}+a_{12} a_{34} b_{21} c_{43}+a_{13} a_{32} b_{21} c_{44} .
\end{aligned}
$$


Then, the local stability of the positive equilibrium $E^{*}$ and the existence of local Hopf bifurcation are discussed in the following four cases.
Case 1. $\tau_{1}=0, \tau_{2}=0$.

Equation (9) at the equilibrium $E^{*}\left(x^{*}, y^{*}, v^{*}, z^{*}\right)$ reduces to

$$
\lambda^{4}+\left(A_{1}+B_{1}+C_{1}\right) \lambda^{3}+\left(A_{2}+B_{2}+C_{2}+D_{1}\right) \lambda^{2}+\left(A_{3}+B_{3}+C_{3}+D_{2}\right) \lambda+A_{4}+B_{4}+C_{4}+D_{3}=0
$$

Let

$$
\begin{aligned}
\left(H_{1}\right) R_{1}> & 1 \\
\left(H_{2}\right) \Delta_{1}= & A_{1}+B_{1}+C_{1}>0 \\
\Delta_{2}= & \left(A_{1}+B_{1}+C_{1}\right)\left(A_{2}+B_{2}+C_{2}+D_{1}\right)-\left(A_{3}+B_{3}+C_{3}+D_{2}\right)>0 \\
\Delta_{3}= & \left(A_{1}+B_{1}+C_{1}\right)\left[\left(A_{2}+B_{2}+C_{2}+D_{1}\right)\left(A_{3}+B_{3}+C_{3}+D_{2}\right)-\left(A_{1}+B_{1}+C_{1}\right)\left(A_{4}+B_{4}+C_{4}+D_{3}\right)\right] \\
& -\left(A_{3}+B_{3}+C_{3}+D_{2}\right)^{2}>0 \\
\Delta_{4}= & A_{4}+B_{4}+C_{4}+D_{3}>0 .
\end{aligned}
$$

From the Routh-Hurwitz criterion, we have the following theorem.
Case 2. $\tau_{1}>0$ and $\tau_{2}=0$.

The characteristic equation of system (2) is

Theorem 1. If $\left(\mathrm{H}_{1}\right)$ and $\left(\mathrm{H}_{2}\right)$ hold, the positive equilibrium $E^{*}$ is locally asymptotically stable for $\tau_{1}=0$ and $\tau_{2}=0$.

$$
\lambda^{4}+\left(A_{1}+C_{1}\right) \lambda^{3}+\left(A_{2}+C_{2}\right) \lambda^{2}+\left(A_{3}+C_{3}\right) \lambda+A_{4}+C_{4}+\left[B_{1} \lambda^{3}+\left(B_{2}+D_{1}\right) \lambda^{2}+\left(B_{3}+D_{2}\right) \lambda+B_{4}+D_{3}\right] e^{-\lambda \tau_{1}}=0
$$

Denote

$$
\begin{aligned}
& F_{1}=A_{1}+C_{1}, \\
& F_{2}=A_{2}+C_{2}, \\
& F_{3}=A_{3}+C_{3}, \\
& F_{4}=A_{4}+C_{4}, \\
& G_{1}=B_{1}, \\
& G_{2}=B_{2}+D_{1}, \\
& G_{3}=B_{3}+D_{2}, \\
& G_{4}=B_{4}+D_{3},
\end{aligned}
$$

then equation (13) is reduced to

$$
P\left(\lambda, \tau_{1}\right)+Q\left(\lambda, \tau_{1}\right) e^{-\lambda \tau_{1}}=0
$$

where $\quad P\left(\lambda, \tau_{1}\right)=\lambda^{4}+F_{1} \lambda^{3}$ $+F_{2} \lambda^{2}+F_{3} \lambda+F_{4}$ and $Q\left(\lambda, \tau_{1}\right)=G_{1} \lambda^{3}+G_{2} \lambda^{2}+G_{3} \lambda+G_{4}$.

Denote $\quad \tau_{\max }=(1 / m) \ln \left(x_{M}\left(k \beta_{1}+u \beta_{2}\right)\left(c k r-k b \beta_{1}-\right.\right.$ $\left.\left.u b \beta_{2}\right) / c k r a u\right)$ and $I=\left\{0 \leq \tau<\tau_{\max }\right.$ : there is $\omega(\tau)>$ 0 such that $F(\omega, \tau)=0\}$. According to the geometrical criterion established by Beretta and Kuang [16], we can easily verify the following conditions for $\omega>0, \tau_{1} \in I$ :

(a) $P\left(0, \tau_{1}\right)+Q\left(0, \tau_{1}\right)=F_{4}+G_{4} \neq 0$.

(b) $P\left(i \omega, \tau_{1}\right)+Q\left(i \omega, \tau_{1}\right)=\omega^{4}-\left(F_{2}+G_{2}\right) \omega^{2}+F_{4}+$ $G_{4}-\left[\left(F_{1}+G_{1}\right) \omega^{3}-\left(F_{3}+G_{3}\right) i \omega+G_{3} \omega\right] i \neq 0$.

(c) $\lim _{|\lambda \longrightarrow \infty|}\left|Q\left(\lambda, \tau_{1}\right) / P\left(\lambda, \tau_{1}\right)\right|=\lim _{|\lambda \longrightarrow \infty|} \mid\left(G_{1} \lambda^{3}+\right.$ $\left.G_{2} \lambda^{2}+G_{3} \lambda+G_{4}\right) /\left.\left(\lambda^{4}+F_{1} \lambda^{3}+F_{2} \lambda^{2}+F_{3} \lambda+F_{4}\right)\right|^{k=1 / \lambda}=$ $\lambda \lim _{|k \longrightarrow 0|} \mid\left(G_{1} k+G_{2} k^{2}+G_{3} k^{3}+G_{4} k^{4}\right) /\left(1+F_{1} k+\right.$ $\left.F_{2} k^{2}+F_{3} k^{3}+F_{4} k^{4}\right) \mid=0$.

(d) $\left|P\left(i \omega, \tau_{1}\right)\right|^{2}=\omega^{8}+\left(F_{1}^{2}-2 F_{2}\right) \quad \omega^{6}+\left(F_{2}^{2}+2 F_{4}-\right.$ $\left.2 F_{1} F_{3}\right) \omega^{4}+\left(F_{3}^{2}-2 F_{2} F_{4}\right) \omega^{2}+F_{4}^{2},\left|Q\left(i \omega, \tau_{1}\right)\right|^{2}=G_{1}^{2}$ $\omega^{6}+\left(G_{2}^{2}-2 G_{1} G_{3}\right) \omega^{4}+\left(G_{3}^{2}-2 G_{2} G_{4}\right) \omega^{2}+G_{4}^{2}, F(\omega$, $\left.\tau_{1}\right)=\left|P\left(i \omega, \tau_{1}\right)\right|^{2}-\left|Q\left(i \omega, \tau_{1}\right)\right|^{2}=\omega^{8}+\left(F_{1}^{2}-2 F_{2}-\right.$ $\left.G_{1}^{2}\right) \omega^{6}+\left(F_{2}^{2}+2 F_{4}-2 F_{1} F_{3}-G_{2}^{2}+2 G_{1} G_{3}\right) \omega^{4}+\left(F_{3}^{2}\right.$ $\left.-2 F_{2} F_{4}-G_{3}^{2}+2 G_{2} G_{4}\right) \omega^{2}+F_{4}^{2}-G_{4}^{2}$.

We can get easily $F\left(\omega, \tau_{1}\right)$ has finite roots.

(f) Each positive root $\omega\left(\tau_{1}\right)$ of $F\left(\omega, \tau_{1}\right)=0$ is continuous and differentiable.

Let $\lambda=i \omega(\omega>0)$ be a root of equation (15) and separate the real and imaginary parts, then we get 


$$
\left\{\begin{array}{l}
\omega^{4}-F_{2} \omega^{2}+F_{4}=-\left(G_{4}-G_{2} \omega^{2}\right) \cos \left(\omega \tau_{1}\right)+\left(G_{1} \omega^{3}-G_{3} \omega\right) \sin \left(\omega \tau_{1}\right), \\
-F_{1} \omega^{3}+F_{3} \omega=\left(G_{4}-G_{2} \omega^{2}\right) \sin \left(\omega \tau_{1}\right)+\left(G_{1} \omega^{3}-G_{3} \omega\right) \cos \left(\omega \tau_{1}\right) .
\end{array}\right.
$$

Then, we can obtain

$$
\begin{aligned}
\cos \left(\omega \tau_{1}\right) & =\frac{\left(\omega^{4}-F_{2} \omega^{2}+F_{4}\right)\left(G_{2} \omega^{2}-G_{4}\right)+\left(F_{3} \omega-F_{1} \omega^{3}\right)\left(G_{1} \omega^{3}-G_{3} \omega\right)}{\left(G_{2} \omega^{2}-G_{4}\right)^{2}+\left(G_{1} \omega^{3}-G_{3} \omega\right)^{2}} \\
\sin \left(\omega \tau_{1}\right) & =\frac{\left(\omega^{4}-F_{2} \omega^{2}+F_{4}\right)\left(G_{1} \omega^{3}-G_{3} \omega\right)-\left(F_{3} \omega-F_{1} \omega^{3}\right)\left(G_{2} \omega^{2}-G_{4}\right)}{\left(G_{2} \omega^{2}-G_{4}\right)^{2}+\left(G_{1} \omega^{3}-G_{3} \omega\right)^{2}}
\end{aligned}
$$

From (16), it follows that

$$
\left(\omega^{4}-F_{2} \omega^{2}+F_{4}\right)^{2}+\left(-F_{1} \omega^{3}+F_{3} \omega\right)^{2}=\left(G_{4}-G_{2} \omega^{2}\right)^{2}+\left(G_{1} \omega^{3}-G_{3} \omega\right)^{2}
$$

which is equivalent to $F\left(\omega, \tau_{1}\right)=0$ for (15). Denote $h=\omega^{2}$, then we have $F\left(\omega, \tau_{1}\right)=G\left(h, \tau_{1}\right)=0$. Therefore, equation (13) has a pair of pure imaginary roots $\pm i \omega_{1}^{*}$ when $\tau_{1}>0$ and $\tau_{2}=0$ if $\omega_{1}^{*}$ is the positive root of $F\left(\omega, \tau_{1}\right)$. Then, we denote $\omega\left(\tau_{1}\right) \tau_{1}=\theta\left(\tau_{1}\right)+2 n \pi$ and $S_{n}\left(\tau_{1}\right)=\tau_{1}-\left(\theta\left(\tau_{1}\right)+2 n \pi\right) / \omega\left(\tau_{1}\right)$. Hence, $i \omega_{1}^{*}$ is a pure imaginary root of equation (13) if and only if $\tau_{1}^{*}$ is a zero of $S_{n}\left(\tau_{1}\right)$. We introduce the following theorem [16].

Theorem 2. Assume that $S_{n}\left(\tau_{1}^{*}\right)=0$ have some positive roots $\tau_{1}^{*} \in I$ for some $n \in N$. Then, a pair of simple pure imaginary roots $\pm i \omega_{1}\left(\tau_{1}^{*}\right)$ exists which crosses the imaginary axis from left to right if $\delta\left(\tau_{1}^{*}\right)>0$ and crosses the imaginary axis from right to left if $\delta\left(\tau_{1}^{*}\right)<0$, where

$$
\delta\left(\tau_{1}^{*}\right)=\operatorname{sign}\left\{F_{\omega_{1}}^{\prime}\left(\omega_{1}\left(\tau_{1}^{*}\right), \tau_{1}^{*}\right)\right\} \operatorname{sign}\left\{\left.\frac{\mathrm{d} S_{n}\left(\tau_{1}\right)}{\mathrm{d} \tau_{1}}\right|_{\tau_{1}=\tau_{1}^{*}}\right\} .
$$

Since $\partial F\left(\omega_{1}, \tau_{1}\right) / \partial \omega_{1}=2 \omega_{1}\left(\partial G\left(\omega_{1}^{2}, \tau_{1}\right) / \partial \omega_{1}\right)$, equation (19) is equivalent to

$$
\operatorname{sign}\left\{\left.\frac{\mathrm{dRe}(\lambda)}{\mathrm{d} \tau}\right|_{\lambda=i \omega_{1}\left(\tau_{1}^{*}\right)}\right\}=\operatorname{sign}\left\{2 \omega_{1}\left(\frac{\partial G}{\partial \omega_{1}}\right)\left(\omega_{1}^{2}, \tau_{1}\right)\right\} \times \operatorname{sign}\left\{\left.\frac{\mathrm{d} S_{n}\left(\tau_{1}\right)}{\mathrm{d} \tau_{1}}\right|_{\tau_{1}=\tau_{1}^{*}}\right\}
$$

Next, we can easily get $S_{n}(0)<0$ and $S_{n}\left(\tau_{1}\right)(n \in N)$ is a monotonic increasing function for all $\tau_{1} \in I$. If $S_{0}$ has no positive root in $I$, then $S_{n}$ also does not have positive root in I. And if $S_{n}\left(\tau_{1}\right)$ has a positive root $\tau_{1} \in I$ for some $n \in N$, then there is at least one positive root which satisfies $\left(\mathrm{d} S_{n}\left(\tau_{1}\right) / \mathrm{d} \tau_{1}\right)\left(\tau_{1}\right)>0$. We introduce the Hopf bifurcation theorem:

Theorem 3. A single parameter system of form $\dot{x}_{t}=F\left(\alpha, x_{t}\right)$ where $F(\alpha, \phi)$ has continuous first and second derivatives for $\alpha \in R, \phi \in C$. Define $L: R \times C \longrightarrow R^{n}$ as $L(\alpha) \psi=F_{\phi}(\alpha, 0) \psi$, where $F_{\phi}(\alpha, 0)$ is the derivation of $F(\alpha, \phi)$ with respect to $\phi$ at $\phi=0$, and define $f(\alpha, \phi)=F(\alpha, \phi)-L(\alpha) \phi$. There are two hypotheses:

(i) The linear differential equation $(L(0))$ has a pure imaginary characteristic root $\lambda_{0}=i \omega_{0} \neq 0$ and none of the other roots are multiples of $i \omega_{0}$ (ii) $\operatorname{Re}\left(\lambda^{\prime}(0)\right) \neq 0$

The hypotheses (i) and (ii) imply that there are nonconstant periodic solutions, and a Hopf bifurcation occurs.

From all above analysis, we have the following theorem.

Theorem 4. Assume that $R_{1}>1$ holds, then

(i) If $S_{0}\left(\tau_{1}\right)$ has no positive root in $I, E^{*}$ is locally asymptotically stable for all $\tau_{1}>0$

(ii) If $S_{0}\left(\tau_{1}\right)$ has at least one positive root in $I$, there exists $\tau_{1}^{*}$ such that $E^{*}$ is locally asymptotically stable for all $\tau_{1} \in\left[0, \tau_{1}^{*}\right]$

(iii) If (ii) holds and $\partial F_{1} / \partial \omega_{1}\left(\omega_{1}^{2}\left(\tau_{1}^{*}\right), \tau_{1}^{*}\right)>0$, a Hopf bifurcation occurs at $E^{*}$ for $\tau_{1}=\tau_{1}^{*}$

Case 3. $\tau_{1}=\tau_{2}=\tau$. 
Denote $P_{0}(\lambda, \tau)=\lambda^{4}+A_{1} \lambda^{3}+A_{2} \lambda^{2}+A_{3} \lambda+A_{4}, P_{1}(\lambda, \tau)$ $=\left(B_{1}+C_{1}\right) \lambda^{3}+\left(B_{2}+C_{2}\right) \lambda^{2}+\left(B_{3}+C_{3}\right) \lambda+\left(B_{4}+C_{4}\right), P_{2}$ $(\lambda, \tau)=D_{1} \lambda^{2}+D_{2} \lambda+D_{3}$, then equation (9) reduces to

$$
P_{0}(\lambda, \tau)+P_{1}(\lambda, \tau) e^{-\lambda \tau}+P_{2}(\lambda, \tau) e^{-2 \lambda \tau}=0 .
$$

Obviously, $P_{l}(\lambda, \tau), l=0,1,2$, is a polynomial with $\lambda$, and its coefficients depend on $\tau$. Next, we discuss the existence of pure imaginary roots of (21) by using the method introduced in [17].

Let $\tau \in\left[0, \tau_{\max }\right)$, we need to verify the following conditions: (a) $\operatorname{deg}\left(P_{0}(\lambda, \tau)\right) \geq \max \left\{\operatorname{deg}\left(P_{1}(\lambda, \tau), \operatorname{deg}\left(P_{2}(\lambda, \tau)\right\}\right.\right.$

(b) $P_{0}(0, \tau)+P_{1}(0, \tau)+P_{2}(0, \tau) \neq 0$

(c) If $\lambda=i \omega, \omega \in R$,

then $P_{0}(i \omega, \tau)+P_{1}(i \omega, \tau)+P_{2}(i \omega, \tau) \neq 0, \tau \in R$

(d) $\lim _{|\lambda| \rightarrow \infty}\left|P_{1}(\lambda, \tau) / P_{0}(\lambda, \tau)\right|+\left|P_{2}(\lambda, \tau) / P_{0}(\lambda, \tau)\right|<1$

(e) $F(\omega, \tau)=R^{2}(\omega, \tau)-T^{2}(\omega, \tau)-S^{2}(\omega, \tau)=0 \quad$ for each $\tau$ has at most a finite number of real zeros where

$$
\begin{aligned}
R(\omega, \tau) & =\left|P_{0}(i \omega, \tau)\right|^{2}-\left|P_{2}(i \omega, \tau)\right|^{2} \\
S(\omega, \tau) & =\operatorname{Re}\left(P_{1}(i \omega, \tau)\right)\left[\operatorname{Im}\left(P_{0}(i \omega, \tau)\right)+\operatorname{Im}\left(P_{2}(i \omega, \tau)\right)\right]-\operatorname{Im}\left(P_{1}(i \omega, \tau)\right)\left[\operatorname{Re}\left(P_{0}(i \omega, \tau)\right)+\operatorname{Re}\left(P_{2}(i \omega, \tau)\right)\right] \\
T(\omega, \tau) & =\operatorname{Re}\left(P_{1}(i \omega, \tau)\right)\left[\operatorname{Re}\left(P_{2}(i \omega, \tau)\right)-\operatorname{Re}\left(P_{0}(i \omega, \tau)\right)\right]+\operatorname{Im}\left(P_{1}(i \omega, \tau)\right)\left[\operatorname{Im}\left(P_{2}(i \omega, \tau)\right)-\operatorname{Im}\left(P_{0}(i \omega, \tau)\right)\right]
\end{aligned}
$$

(f) Each positive root $\omega(\tau)$ of $F(\omega, \tau)=0$ is continuous and differentiable in $\tau$ whenever it exists

Next, we verify the above six conditions.

(a) It is obviously satisfied.

(b) $P_{0}(0, \tau)+P_{1}(0, \tau)+P_{2}(0, \tau)=A_{4}+B_{4}+C_{4}+D_{3}$ according to $\left(H_{2}\right), P_{0}(0, \tau)+P_{1}(0, \tau)+P_{2}(0, \tau)>0$.

(c) $P_{0}(i \omega, \tau)+P_{1}(i \omega, \tau)+P_{2}(i \omega, \tau)=\omega^{4}-\left(A_{2}+B_{2}+\right.$ $\left.C_{2}+D_{1}\right) \omega^{2}+A_{4}+B_{4}+C_{4}+D_{3}+i \omega\left[-\left(A_{1}+B_{1}+\right.\right.$ $\left.\left.C_{1}\right) \omega^{2}+A_{3}+B_{3}+C_{3}+D_{2}\right] \neq 0$.

(d) $\lim _{|\lambda| \longrightarrow \infty}\left|P_{1}(\lambda, \tau) / P_{0}(\lambda, \tau)\right|+\left|P_{2}(\lambda, \tau) / P_{0}(\lambda, \tau)\right|=$ $\lim _{|\lambda| \rightarrow \infty} \mid\left[\left(B_{1}+C_{1}\right) \lambda^{3}+\left(B_{2}+C_{2}\right) \lambda^{2}+\left(B_{3}+C_{3}\right) \lambda+\right.$ $\left.B_{4}+C_{4}\right] /\left(\lambda^{4}+A_{1} \lambda^{3}+A_{2} \lambda^{2}+A_{3} \lambda+A_{4}|+|\left(D_{1} \lambda^{2}+D_{2}\right.\right.$ $\left.\left.\lambda+D_{3}\right) /\left(\lambda^{4}+A_{1} \lambda^{3}+A_{2} \lambda^{2}+A_{3} \lambda+A_{4}\right) \mid\right)=0$.

(e) $P_{0}(i \omega, \tau)=\left(\omega^{4}-A_{2} \omega^{2}+A_{4}\right)+\left(A_{3} \omega-A_{1} \omega^{3}\right) i, P_{1}$ $(i \omega, \tau)=\left[-\left(B_{2}+C_{2}\right) \omega^{2}+\left(B_{4}+C_{4}\right)\right]+\left[-\left(B_{1}+C_{1}\right)\right.$
$\omega^{3}+\left(B_{3}+C_{3} \omega\right] i, \quad P_{2}(i \omega, \tau)=-\left(D_{1} \omega^{2}+D_{3}\right)+D_{2}$ $\omega i, R(\omega, \tau)=\left(\omega^{4}-A_{2} \omega^{2}+A_{4}\right)^{2}+\left(A_{3} \omega-A_{1} \omega^{3}\right)^{2}-$ $\left(D_{1} \omega^{2}+D_{3}\right)^{2}+D_{2}^{2} \omega^{2}, S(\omega, \tau)=\left[-\left(B_{2}+C_{2}\right) \omega^{2}+\right.$ $\left.\left(B_{4}+C_{4}\right)\right]\left(A_{3} \omega-A_{1} \omega^{3}+D_{2} \omega\right)-\left[-\left(B_{1}+C_{1}\right) \omega^{3}+\right.$ $\left.\left(B_{3}+C_{3}\right) \omega\right]\left(\omega^{4}-A_{2} \omega^{2}+A_{4}-D_{1} \omega^{2}+D_{3}\right), T(\omega, \tau)$ $=\left[-\left(B_{2}+C_{2}\right) \omega^{2}+B_{4}+C_{4}\right]\left[-\left(D_{1} \omega^{2}+D_{3}\right)-\left(\omega^{4}\right.\right.$ $\left.\left.-A_{2} \omega^{2}+A_{4}\right)\right]+\left(A_{3} \omega-A_{1} \omega^{3}\right)\left[D_{2} \omega-\left(A_{3} \omega-A_{1} \omega^{3}\right)\right]$.

Substituting $R(\omega, \tau), S(\omega, \tau), T(\omega, \tau)$ into $F(\omega, \tau)$ $=R^{2}(\omega, \tau)-T^{2}(\omega, \tau)-S^{2}(\omega, \tau)=0$, then we get this condition holds.

(f) The implicit function theorem shows that this condition is true.

Assuming that $\lambda=i \omega(\omega>0)$ is a root of equation (13), then we have

$$
\begin{aligned}
& \left(\omega^{4}-A_{1} i \omega^{3}-A_{2} \omega^{2}+A_{3} i \omega+A_{4}\right)(\cos (\omega \tau)+i \sin (\omega \tau))-\left(B_{1}+C_{1}\right) i \omega^{3}-\left(B_{2}+C_{2}\right) \omega^{2}+\left(B_{3}+C_{3}\right) i \omega+\left(B_{4}+C_{4}\right) \\
& +\left(-D_{1} \omega^{2}+D_{2} i \omega+D_{3}\right)(\cos (\omega \tau)-i \sin (\omega \tau))=0 .
\end{aligned}
$$

Separating the real and imaginary parts, we have

$$
\left\{\begin{array}{l}
\cos (\omega \tau)\left[\omega^{4}-\left(A_{2}+D_{1}\right) \omega^{2}+A_{4}+D_{3}\right]+\sin (\omega \tau)\left[A_{1} \omega^{3}+\left(D_{2}-A_{3}\right) \omega\right]=\left(B_{2}+C_{2}\right) \omega^{2}-\left(B_{4}+C_{4}\right), \\
\cos (\omega \tau)\left[-A_{1} \omega^{3}+\left(A_{3}+D_{2}\right) \omega\right]+\sin (\omega \tau)\left[\omega^{4}-\left(A_{2}-D_{1}\right) \omega^{2}+A_{4}-D_{3}\right]=\left(B_{1}+C_{1}\right) \omega^{3}-\left(B_{3}+C_{3}\right) \omega .
\end{array}\right.
$$


Therefore, $\omega=\omega(\tau)>0$ needs to satisfy the following equations:

$$
\left\{\begin{array}{l}
\cos (\omega \tau)=\frac{l_{5} \omega^{6}+l_{6} \omega^{4}+l_{7} \omega^{2}+l_{8}}{\omega^{8}+k_{1} \omega^{6}+k_{2} \omega^{4}+k_{3} \omega^{2}+k_{4}}=\frac{T(\omega, \tau)}{R(\omega, \tau)}, \\
\sin (\omega \tau)=\frac{l_{1} \omega^{7}+l_{2} \omega^{5}+l_{3} \omega^{3}+l_{4} \omega}{\omega^{8}+k_{1} \omega^{6}+k_{2} \omega^{4}+k_{3} \omega^{2}+k_{4}}=\frac{S(\omega, \tau)}{R(\omega, \tau)},
\end{array}\right.
$$

where

$$
\begin{aligned}
k_{1}= & A_{1}^{2}-2 A_{2}, \\
k_{2}= & 2 A_{4}+A_{2}^{2}-D_{1}^{2}-2 A_{1} A_{3}, \\
k_{3}= & A_{3}^{2}-D_{2}^{2}-2 A_{2} A_{4}+2 D_{1} D_{3}, \\
k_{4}= & A_{4}^{2}-D_{3}^{2}, \\
l_{1}= & \left(B_{1}+C_{1}\right), \\
l_{2}= & A_{1}\left(B_{2}+C_{2}\right)-\left(B_{3}+C_{3}\right)-\left(B_{1}+C_{1}\right)\left(A_{2}+D_{1}\right), \\
l_{3}= & \left(B_{1}+C_{1}\right)\left(A_{4}+D_{3}\right)+\left(B_{3}+C_{3}\right)\left(A_{2}+D_{1}\right)-\left(B_{2}+C_{2}\right) \\
& \left(A_{3}+D_{2}\right)-A_{1} B_{4}, \\
l_{4}= & \left(B_{4}+C_{4}\right)\left(A_{3}+D_{2}\right)-\left(B_{3}+C_{3}\right)\left(A_{4}+D_{3}\right), \\
l_{5}= & \left(B_{2}+C_{2}\right)-A_{1}\left(B_{1}+C_{1}\right), \\
l_{6}= & -\left(B_{4}+C_{4}\right)+\left(B_{1}+C_{1}\right)\left(A_{3}-D_{2}\right)-\left(B_{2}+C_{2}\right)\left(A_{2}-D_{1}\right) \\
& +A_{1}\left(B_{3}+C_{3}\right), \\
l_{7}= & \left(B_{2}+C_{2}\right)\left(A_{4}-D_{3}\right)-\left(B_{3}+C_{3}\right)\left(A_{3}-D_{2}\right)+B_{4}\left(A_{2}-D_{1}\right), \\
l_{8}= & -\left(B_{4}+C_{4}\right)\left(A_{4}-D_{3}\right) .
\end{aligned}
$$

Equation (25) is equivalent to the condition that $\omega=$ $\omega(\tau)>0$ is the root of $F(\omega, \tau)=R^{2}(\omega, \tau)-T^{2}(\omega, \tau)-$ $S^{2}(\omega, \tau)=0$.
Let $I=\{\tau$ : there is $\omega(\tau)>0$ such that $F(\omega, \tau)=$ $0\} \cup\left[0, \tau_{\max }\right)$, then we denote $S_{n}(\tau)=\tau-(\theta(\tau)+2 n \pi) /$ $\omega(\tau), \tau \in I, n \in N_{0}$, where $\theta(\tau) \in[0,2 \pi] . \omega(\tau) \tau=\theta(\tau)+2 n \pi$. Hence, $i \omega^{*}\left(\omega^{*}=\omega\left(\tau^{*}\right)>0\right)$ is the pure imaginary root of $(21)$ and is a necessary and sufficient condition for $\tau^{*}$ being the root of $S_{n}(\tau)=0$. We need to introduce the following theorem [18].

Theorem 5. Assume that $S_{n}(\tau)=0$ has some positive roots $\tau^{*} \in I$ for some $n \in N_{0}$. Then, a pair of simple pure imaginary roots $\pm i \omega\left(\tau^{*}\right)$ exists when $\tau=\tau^{*}$ which crosses the imaginary axis from left to right if $\delta\left(\tau^{*}\right)>0$ and crosses the imaginary axis from right to left if $\delta\left(\tau^{*}\right)<0$, where

$$
\begin{aligned}
\delta\left(\tau^{*}\right) & =\operatorname{sign}\left\{\left.\frac{\mathrm{dRe}(\lambda)}{\mathrm{d} \tau}\right|_{\lambda=i \omega\left(\tau^{*}\right)}\right\} \\
& =\operatorname{sign}\left\{\left.\left[R(\omega(\tau), \tau) F_{\omega}^{\prime}(\omega(\tau), \tau)\right]\right|_{\tau=\tau^{*}}\right\} \times \operatorname{sign}\left\{S_{n}^{\prime}\left(\tau^{*}\right)\right\} .
\end{aligned}
$$

From the above discussion, the following theorem can be obtained.

Theorem 6. Assume that $R_{1}>1$ holds, then

(i) If $I=\phi$ or $I \neq \phi, S_{n}(\tau)=0$ has no positive root $\tau^{*} \in I$ and $E^{*}$ is locally asymptotically stable for all $\tau \in\left[0, \tau_{\max }\right)$

(ii) If $I \neq \phi, S_{n}(\tau)=0$ has at least one positive root $\tau^{*} \in I$ and $\delta\left(\tau^{*}\right) \neq 0, E^{*}$ is locally asymptotically stable when $\tau \in\left[0, \tau^{*}\right)$, and a Hopf bifurcation occurs at $E^{*}$ for $\tau=\tau^{*}$

Case 4. $\tau_{1} \in\left[0, \tau_{1}^{*}\right), \tau_{2}>0, \tau_{1} \neq \tau_{2}$.

In this case, we consider $\tau_{2}$ as a parameter and $\tau_{1}=\tau_{10}^{*} \in\left[0, \tau_{\max }\right)$. Let $\lambda=i \omega(\omega>0)$ be a root of $(9)$, then we get

$$
\left\{\begin{array}{l}
\omega^{4}-A_{2} \omega^{2}+A_{4}+\left(B_{4}-B_{2} \omega^{2}\right) \cos \left(\omega \tau_{1}\right)+\left(B_{3} \omega-B_{1} \omega^{3}\right) \sin \left(\omega \tau_{1}\right)=\left[C_{2} \omega^{2}-C_{4}-\left(D_{3}-D_{1} \omega^{2}\right) \cos \left(\omega \tau_{1}\right)-D_{2} \omega \sin \left(\omega \tau_{1}\right)\right] \\
\cos \left(\omega \tau_{2}\right)+\left[C_{1} \omega^{3}-C_{3} \omega+\left(D_{3}-D_{1} \omega^{2}\right) \sin \left(\omega \tau_{1}\right)-D_{2} \omega \cos \left(\omega \tau_{1}\right)\right] \sin \left(\omega \tau_{2}\right) \\
-A_{1} \omega^{3}+A_{3} \omega-\left(B_{4}-B_{2} \omega^{2}\right) \sin \left(\omega \tau_{1}\right)+\left(B_{3} \omega-B_{1} \omega^{3}\right) \cos \left(\omega \tau_{1}\right)=\left[C_{4}-C_{2} \omega^{2}+\left(D_{3}-D_{1} \omega^{2}\right) \cos \left(\omega \tau_{1}\right)+D_{2} \omega \sin \left(\omega \tau_{1}\right)\right] \\
\sin \left(\omega \tau_{2}\right)+\left[C_{1} \omega^{3}-C_{3} \omega+\left(D_{3}-D_{1} \omega^{2}\right) \sin \left(\omega \tau_{1}\right)-D_{2} \omega \cos \left(\omega \tau_{1}\right)\right] \cos \left(\omega \tau_{2}\right) .
\end{array}\right.
$$

From (29), we have

$$
\cos \left(\omega \tau_{2}\right)=\frac{h_{1} \omega^{6}+h_{2} \omega^{5}+h_{3} \omega^{4}+h_{4} \omega^{3}+h_{5} \omega^{2}+h_{6} \omega+h_{7}}{f_{1} \omega^{6}+f_{2} \omega^{5}+f_{3} \omega^{4}+f_{4} \omega^{3}+f_{5} \omega^{2}+f_{6} \omega+f_{7}},
$$


where

$$
\begin{aligned}
& h_{1}=C_{2}-A_{1} C_{1}+\left(D_{1}-B_{1} C_{1}\right) \cos \left(\omega \tau_{1}\right) \\
& h_{2}=\left(A_{1} D_{1}-D_{2}-B_{1} C_{2}+B_{2} C_{1}\right) \sin \left(\omega \tau_{1}\right), \\
& h_{3}=A_{1} C_{3}-C_{4}-A_{2} C_{2}+A_{3} C_{1}+B_{1} D_{2}-B_{2} D_{1}+\left(-D_{3}+A_{1} D_{2}-A_{2} D_{1}+B_{1} C_{3}-B_{2} C_{2}+B_{3} C_{1}\right) \cos \left(\omega \tau_{1}\right), \\
& h_{4}=\left(A_{2} D_{2}-A_{1} D_{3}-A_{3} D_{1}+B_{1} C_{4}-B_{2} C_{3}+B_{3} C_{2}-B_{4} C_{4}\right) \sin \left(\omega \tau_{1}\right), \\
& h_{5}=A_{2} C_{4}-A_{3} C_{3}+A_{4} C_{2}+B_{2} D_{3}-B_{3} D_{2}+B_{4} D_{1}+\left(A_{2} D_{3}-A_{3} D_{2}+A_{4} D_{1}+B_{2} C_{4}-B_{3} C_{3}+B_{4} C_{2}\right) \cos \left(\omega \tau_{1}\right), \\
& h_{6}=\left(A_{3} D_{3}-A_{4} D_{2}-B_{3} C_{4}+B_{4} C_{3}\right) \sin \left(\omega \tau_{1}\right) \\
& h_{7}=-A_{4} C_{4}-B_{4} D_{3}-\left(A_{4} D_{3}+B_{4} C_{4}\right) \cos \left(\omega \tau_{1}\right) \\
& f_{1}=C_{1}^{2} \\
& f_{2}=-2 C_{1} D_{1} \sin \left(\omega \tau_{1}\right) \\
& f_{3}=C_{2}^{2}-2 C_{1} C_{3}+D_{1}^{2}+\left(2 C_{2} D_{1}-2 C_{1} D_{2}\right) \cos \left(\omega \tau_{1}\right), \\
& f_{4}=\left(2 C_{1} D_{3}-2 C_{2} D_{2}+2 C_{3} D_{1}\right) \sin \left(\omega \tau_{1}\right), \\
& f_{5}=-2 C_{2} C_{4}+C_{3}^{2}+D_{2}^{2}-2 D_{1} D_{3}+\left(2 C_{3} D_{2}-2 C_{2} D_{3}-2 C_{4} D_{1}\right) \cos \left(\omega \tau_{1}\right), \\
& f_{6}=\left(2 C_{4} D_{2}-2 C_{3} D_{3}\right) \sin \left(\omega \tau_{1}\right), \\
& f_{7}=C_{4}^{2}+D_{3}^{2}+2 C_{4} D_{3} \cos \left(\omega \tau_{1}\right) .
\end{aligned}
$$

We can also get where

$$
\begin{aligned}
F(\omega) \triangleq & \omega^{8}+I_{1} \omega^{6}+I_{2} \omega^{4}+I_{3} \omega^{2}+I_{4}+I_{5} \sin \left(\omega \tau_{1}\right) \\
& +I_{6} \cos \left(\omega \tau_{1}\right)=0,
\end{aligned}
$$

$$
\begin{aligned}
I_{1}= & -2 A_{2}+A_{1}^{2}-C_{1}^{2}+B_{1}^{2}, \\
I_{2}= & 2 A_{4}+B_{2}^{2}-D_{1}^{2}+A_{2}^{2}-C_{2}^{2}-2 A_{1} A_{3}-2 B_{1} B_{3}+2 C_{1} C_{3}, \\
I_{3}= & B_{3}^{2}-D_{2}^{2}+A_{3}^{2}-C_{3}^{2}-2 A_{2} A_{4}-2 B_{2} B_{4}+2 C_{2} C_{4}+2 D_{1} D_{3}, \\
I_{4}= & B_{4}^{2}-D_{3}^{2}+A_{4}^{2}-C_{4}^{2}, \\
I_{5}= & -2 B_{1} \omega^{7}+\left(2 B_{3}-2 A_{1} B_{2}+2 A_{2} B_{1}+2 C_{1} D_{1}\right) \omega^{5}+\left(2 A_{1} B_{4}-2 A_{2} B_{3}+2 A_{3} B_{2}-2 A_{4} B_{1}+2 C_{2} D_{2}-2 C_{3} D_{1}-2 C_{1} D_{3}\right) \omega^{3} \\
& +\left(2 A_{4} B_{3}-2 A_{3} B_{4}+2 C_{3} D_{3}-2 C_{4} D_{2}\right) \omega, \\
I_{6}= & \left(2 B_{2}+2 A_{1} B_{1}\right) \omega^{6}+\left(2 B_{4}+2 A_{2} B_{2}-2 C_{2} D_{1}-2 A_{1} B_{3}-2 A_{3} B_{1}+2 C_{1} D_{2}\right) \omega^{4} \\
& +\left(2 A_{3} B_{3}-2 C_{3} D_{2}-2 A_{2} B_{4}-2 A_{4} B_{2}+2 C_{4} D_{1}+2 C_{2} D_{3}\right) \omega^{2}+2 A_{4} B_{4}-2 C_{4} D_{3} .
\end{aligned}
$$

If $B_{4}^{2}-D_{3}^{2}+A_{4}^{2}-C_{4}^{2}-2 A_{4} B_{4}-2 C_{4} D_{3}<0, \quad$ then $F(0)<0$ and $\lim _{\omega \rightarrow \infty} F(\omega) \longrightarrow+\infty$. We obtain that (31) has at least one positive real root. Suppose that there are eight positive real roots of (31) which are $\omega_{1}^{*}, \omega_{2}^{*}, \ldots, \omega_{8}^{*}$. According to (29), we have

$$
\tau_{2 k}^{*(j)}=\frac{1}{\omega_{k}^{*}}\left\{\arccos \left(\frac{h_{1} \omega^{6}+h_{2} \omega^{5}+h_{3} \omega^{4}+h_{4} \omega^{3}+h_{5} \omega^{2}+h_{6} \omega+h_{7}}{f_{1} \omega^{6}+f_{2} \omega^{5}+f_{3} \omega^{4}+f_{4} \omega^{3}+f_{5} \omega^{2}+f_{6} \omega+f_{7}}\right)+2 j \pi\right\},
$$


where $k=1, \ldots, 8, j=0,1,2, \ldots$. Define

$$
\begin{aligned}
& \tau_{20}^{*}=\tau_{2 k 0}^{*}(0)=\min _{k \in 1, \ldots, 8}\left\{\tau_{2 k 0}^{*}(0)\right\}, \\
& \omega_{20}^{*}=\omega_{2 k_{0}}^{*} .
\end{aligned}
$$

Taking the derivative of (9) with respect to $\tau_{2}$, we can get

$$
\left[\frac{\mathrm{d} \lambda}{\mathrm{d} \tau_{2}}\right]^{-1}=\frac{1}{\lambda} \frac{Q_{1}+Q_{2} e^{-\lambda \tau_{1}}+Q_{3} e^{-\lambda \tau_{2}}+Q_{4} e^{-\lambda\left(\tau_{1}+\tau_{2}\right)}}{Q_{5} e^{-\lambda \tau_{2}}+Q_{6} e^{-\lambda\left(\tau_{1}+\tau_{2}\right)}}-\frac{\tau_{2}}{\lambda},
$$

where

$$
\begin{aligned}
& Q_{1}=4 \lambda^{3}+3 A_{1} \lambda^{2}+2 A_{2} \lambda+A_{3}, \\
& Q_{2}=3 B_{1} \lambda^{2}+2 B_{2} \lambda+B_{3}-\tau_{1}\left(B_{1} \lambda^{3}+B_{2} \lambda^{2}+B_{3} \lambda+B_{4}\right), \\
& Q_{3}=3 C_{1} \lambda^{2}+2 C_{2} \lambda+C_{3}, \\
& Q_{4}=3 C_{1} \lambda^{2}+2 C_{2} \lambda+C_{3}-\tau_{1}\left(D_{1} \lambda^{2}+D_{2} \lambda+D_{3}\right), \\
& Q_{5}=C_{1} \lambda^{3}+C_{2} \lambda^{2}+C_{3} \lambda+C_{4}, \\
& Q_{6}=D_{1} \lambda^{2}+D_{2} \lambda+D_{3} .
\end{aligned}
$$

From (35), it follows that

$$
\operatorname{Re}\left[\frac{\mathrm{d} \lambda \tau_{2 k}^{*}(j)}{\mathrm{d} \tau_{2}}\right]_{\lambda=i \omega_{2 k}^{*}}^{-1}=\frac{R_{1} R_{3}+R_{2} R_{4}}{R_{3}^{2}+R_{4}^{2}},
$$

where 
$y^{*}, u_{3}=v-v^{*}, u_{4}=z-z^{*}, t=t / \tau_{2}, \tau_{2}=\tau_{20}^{*}+\mu, \quad$ and $\mu \epsilon$ $R^{4}$. System (2) can be written as an FDE in $C=C\left([-1,0], R^{4}\right)$ as the following form:

$$
\dot{u}(t)=L_{\mu}\left(u_{t}\right)+f\left(\mu, u_{t}\right),
$$

where $u(t)=(x(t), y(t), v(t), z(t))^{T} \in R^{4}$, and $L_{\mu}: C \longrightarrow$ $R^{4}, f: R \times C \longrightarrow R^{4}$ are shown as follows:

$$
\begin{aligned}
& L_{\mu}(\phi)=\left(\tau_{20}^{*}+\mu\right) A\left(\begin{array}{c}
\phi_{1}(0) \\
\phi_{2}(0) \\
\phi_{3}(0) \\
\phi_{4}(0)
\end{array}\right)+\left(\tau_{20}^{*}+\mu\right) B\left(\begin{array}{c}
\phi_{1}\left(-\frac{\tau_{1}}{\tau_{20}^{*}}\right) \\
\phi_{2}\left(-\frac{\tau_{1}}{\tau_{20}^{*}}\right) \\
\phi_{3}\left(-\frac{\tau_{1}}{\tau_{20}^{*}}\right) \\
\phi_{4}\left(-\frac{\tau_{1}}{\tau_{20}^{*}}\right)
\end{array}\right)+\left(\tau_{20}^{*}+\mu\right) C\left(\begin{array}{c}
\phi_{1}(-1) \\
\phi_{2}(-1) \\
\phi_{3}(-1) \\
\phi_{4}(-1)
\end{array}\right), \\
& f(\mu, \phi)=\left(\tau_{20}^{*}+\mu\right)\left(\begin{array}{l}
f_{1} \\
f_{2} \\
f_{3} \\
f_{4}
\end{array}\right) \text {, }
\end{aligned}
$$

where

$$
\begin{aligned}
f_{1}= & -\frac{r \phi_{1}(0)^{2}}{x_{M}}-\beta_{1} \phi_{1}(0) \phi_{3}(0)-\beta_{2} \phi_{1}(0) \phi_{2}(0)+\ldots, \\
f_{2}= & \beta_{1} e^{-m \tau_{1}} \phi_{1}\left(-\frac{\tau_{1}}{\tau_{20}^{*}}\right) \phi_{3}\left(-\frac{\tau_{1}}{\tau_{20}^{*}}\right) \\
& -\beta_{2} e^{-m \tau_{1}} \phi_{1}\left(-\frac{\tau_{1}}{\tau_{20}^{*}}\right) \phi_{2}\left(-\frac{\tau_{1}}{\tau_{20}^{*}}\right)+\ldots, \\
f_{3}= & -p \phi_{3}(0) \phi_{4}(0)+\ldots, \\
f_{4}= & c \phi_{3}(-1) \phi_{4}(-1)+\ldots,
\end{aligned}
$$

and $\phi=\left(\phi_{1}, \phi_{2}, \phi_{3}, \phi_{4}\right) \in R^{4}$.

By the Riesz representation theorem, there exists a function $\eta(\theta, \mu)$ of bounded variation for $\theta \in[-1,0]$, such that

$$
L_{\mu}(\phi)=\int_{-1}^{0} \mathrm{~d} \eta(\theta, \mu) \phi(\theta), \quad \phi \in\left([-1,0], R^{4}\right) .
$$

Next, we choose

$$
\eta(\theta, \mu)=\left(\tau_{20}^{*}+\mu\right)\left[A \delta(\theta)+C \delta(\theta+1)+B \delta\left(\theta+\frac{\tau_{1}}{\tau_{20}^{*}}\right)\right],
$$

where $\delta$ is the Dirac delta function. Define

$$
A(\mu) \phi= \begin{cases}\frac{\mathrm{d} \phi(\theta)}{\mathrm{d} \theta}, & \theta \in[-1,0), \\ \int_{-1}^{0} \mathrm{~d} \eta(s, \mu) \phi(s), & \theta=0,\end{cases}
$$

$R(\mu) \phi= \begin{cases}0, & \theta \in[-1,0), \\ f(\mu, \phi), & \theta=0,\end{cases}$

where $\phi \in C^{1}\left([-1,0], R^{4}\right)$. When $\theta=0$, equation (39) becomes $\dot{u}_{t}=A(\mu) u_{t}+R(\mu) u_{t}$, where $u_{t}(\theta)=u(t+\theta)=$ $(x(t+\theta), y(t+\theta), v(t+\theta), z(t+\theta)), \theta \in[-1,0)$. Define

$$
A^{*} \psi(s)= \begin{cases}-\frac{\mathrm{d} \psi(s)}{\mathrm{d} s}, & s \in(0,1], \\ \int_{-1}^{0} \mathrm{~d} \eta^{T}(t, 0) \psi(-t), & s=0,\end{cases}
$$

$\langle\psi(s), \phi(\theta)\rangle=\bar{\psi}(0) \phi(0)-\int_{-1}^{0} \int_{\xi=0}^{\theta} \bar{\psi}(\xi-\theta) \mathrm{d} \eta(\theta) \phi(\xi) \mathrm{d} \xi$,

where $\eta(\theta)=\eta(\theta, 0)$. $A$ and $A^{*}$ are adjoint operators. Let $A=A(0)$, we know that $\pm i \omega_{20}^{*} \tau_{20}^{*}$ are eigenvalues of both $A$ and $A^{*}$. Assume that $q(\theta)=(1, \alpha, \beta, \gamma)^{T} e^{i \theta \omega_{20}^{*} \tau_{20}^{*}}$ is the eigenvector of $A$ corresponding to $i \omega_{20}^{*} \tau_{20}^{*}$. Then, we have 


$$
\tau_{20}^{*}\left(\begin{array}{cccc}
i \omega_{20}^{*}-a_{11} & -a_{12} & -a_{13} & 0 \\
-b_{21} e^{-i \omega_{20}^{*} \tau_{1}} & i \omega_{20}^{*}-a_{22}-b_{22} e^{-i \omega_{20}^{*} \tau_{1}} & -b_{23} e^{-i \omega_{20}^{*} \tau_{1}} & 0 \\
0 & -a_{32} & i \omega_{20}^{*}-a_{33} & -a_{34} \\
0 & 0 & -c_{43} e^{-i \omega_{20}^{*} \tau_{20}^{*}} & i \omega_{20}^{*}-a_{44}-c_{44} e^{-i \omega_{20}^{*} \tau_{20}^{*}}
\end{array}\right)\left(\begin{array}{l}
1 \\
\alpha \\
\beta \\
\gamma
\end{array}\right)=\left(\begin{array}{l}
0 \\
0 \\
0 \\
0
\end{array}\right)
$$

which yields

$$
q(0)=\left(1, \frac{b_{23} e^{-i \omega_{20}^{*} \tau_{1}}\left(i \omega_{20}^{*}-a_{11}\right)+b_{21} a_{13} e^{-i \omega_{20}^{*} \tau_{1}}}{a_{12} b_{23} e^{-i \omega_{20}^{*} \tau_{1}}+a_{13}\left(i \omega_{20}^{*}-a_{22}-b_{22} e^{-i \omega_{20}^{*} \tau_{1}}\right)}, \frac{i \omega_{20}^{*}-a_{11}-a_{12} \alpha}{a_{13}}, \frac{c_{43} \beta e^{-i \omega_{20}^{*} \tau_{20}^{*}}}{i \omega_{20}^{*}-a_{44}-c_{44} e^{-i \omega_{20}^{*} \tau_{20}^{*}}}\right)^{T} .
$$

Similarly, it can be verified that $q^{*}(s)=D\left(1, \alpha^{*}\right.$, $\left.\beta^{*}, \gamma^{*}\right) e^{i s \omega_{20}^{*} \tau_{20}^{*}}$ is the eigenvector of $A^{*}$ corresponding to $-i \omega_{20}^{*} \tau_{20}^{*}$, where

$$
\begin{aligned}
& \alpha^{*}=-\frac{i \omega_{20}^{*}+a_{11}}{b_{21} e^{i \omega_{20}^{*} \tau_{1}}}, \\
& \beta^{*}=-\frac{a_{12}+\left(i \omega_{20}^{*}+a_{22}+b_{22} e^{i \omega_{20}^{*} \tau_{1}}\right) \alpha^{*}}{a_{32}}, \\
& \gamma^{*}=-\frac{a_{34} \beta^{*}}{i \omega_{20}^{*}+a_{44}+c_{44} e^{i \omega_{20}^{*} \tau_{20}^{*}}} .
\end{aligned}
$$

$$
\begin{aligned}
\left\langle q^{*}(s), q(\theta)\right\rangle= & \bar{D}\left(1, \overline{\alpha^{*}}, \overline{\beta^{*}}, \overline{\gamma^{*}}\right)(1, \alpha, \beta, \gamma)^{T} \\
& -\int_{-1}^{0} \int_{\xi=0}^{\theta} \bar{D}\left(1, \overline{\alpha^{*}}, \overline{\beta^{*}}, \overline{\gamma^{*}}\right) e^{-i(\xi-\theta) \omega_{20}^{*} \tau_{20}^{*}} \mathrm{~d} \eta(\theta)(1, \alpha, \beta, \gamma)^{T} e^{i \xi \omega_{20}^{*} \tau_{20}^{*}} \mathrm{~d} \xi \\
= & \bar{D}\left[1+\alpha \overline{\alpha^{*}}+\beta \overline{\beta^{*}}+\gamma \overline{\gamma^{*}}-\int_{-1}^{0}\left(1, \overline{\alpha^{*}}, \overline{\beta^{*}}, \overline{\gamma^{*}}\right) \theta e^{i \theta \omega_{20}^{*} \tau_{20}^{*}} \mathrm{~d} \eta(\theta)(1, \alpha, \beta, \gamma)^{T}\right] \\
= & \bar{D}\left[1+\alpha \overline{\alpha^{*}}+\beta \overline{\beta^{*}}+\gamma \overline{\gamma^{*}}-\tau_{20}^{*} e^{-i \omega_{20}^{*} \tau_{1}} e^{-m \tau_{1}}\left[\bar{\alpha}^{*}\left(\beta_{1} v^{*}+\beta_{2} y^{*}\right)+\alpha \bar{\alpha}^{*} x^{*}+\beta \bar{\alpha}^{*} x^{*}\right]\right. \\
& \left.-\tau_{20}^{*} e^{-i \omega_{20} \tau_{20}^{*}}\left(\beta \bar{\gamma}^{*} c z^{*}+\gamma \bar{\gamma}^{*} c v^{*}\right)\right] .
\end{aligned}
$$

Thus, we have

$$
\begin{aligned}
\bar{D}= & {\left[1+\alpha \overline{\alpha^{*}}+\beta \overline{\beta^{*}}+\gamma \overline{\gamma^{*}}-\tau_{20}^{*} e^{-i \omega_{20}^{*} \tau_{1}} e^{-m \tau_{1}}\right.} \\
& \cdot\left[\bar{\alpha}^{*}\left(\beta_{1} v^{*}+\beta_{2} y^{*}\right)+\alpha \bar{\alpha}^{*} x^{*}+\beta \bar{\alpha}^{*} x^{*}\right] \\
& \left.-\tau_{20}^{*} e^{-i \omega_{20}^{*} \tau_{20}^{*}}\left(\beta \bar{\gamma}^{*} c z^{*}+\gamma \bar{\gamma}^{*} c v^{*}\right)\right]^{-1},
\end{aligned}
$$

such that $\left\langle q^{*}(s), q(\theta)\right\rangle=1$.

Then, we compute the coordinates that describe the center manifold $C_{0}$ at $\mu=0$. Let $u_{t}$ be the solution of equation (39) at $\mu=0$ and define

$$
\begin{aligned}
z(t) & =\left\langle q^{*}, u_{t}\right\rangle, \\
W(t, \theta) & =u_{t}(\theta)-2 \operatorname{Re}\{z(t) q(\theta)\} .
\end{aligned}
$$

On the center manifold $C_{0}$, we have

$$
W(t, \theta)=W(z(t), \bar{z}(t), \theta)=W_{20}(0) \frac{z^{2}}{2}+W_{11}(\theta) z \bar{z}
$$

$$
+W_{02}(\theta) \frac{\bar{z}^{2}}{2}+W_{30}(\theta) \frac{z^{3}}{6}+\cdots
$$

where $z$ and $\bar{z}$ are local coordinates for center manifold $C_{0}$ in the direction of $q$ and $\bar{q}$.

When $\theta=0$, we have

$$
\dot{z}(t)=i \omega_{20}^{*} \tau_{20}^{*} z+\bar{q}^{*}(0) f_{0}(z, \bar{z}) \triangleq i \omega_{20}^{*} \tau_{20}^{*} z+g(z, \bar{z})
$$

where 


$$
\begin{aligned}
g(z, \bar{z})= & \overline{q^{*}}(0) f_{0}(z, \bar{z})=g_{20} \frac{z^{2}}{2}+g_{11} z \bar{z}+g_{02} \frac{\bar{z}^{2}}{2} \\
& +g_{21} \frac{\bar{z} z^{2}}{2}+\cdots
\end{aligned}
$$

From (52), we have $u_{t}(\theta)=W(t, \theta)+z q(\theta)+\bar{z} \bar{q}(\theta)$. Comparing the coefficients with (54), we get

Then,

$$
\begin{aligned}
g(z, \bar{z})= & \overline{q^{*}}(0) f_{0}(z, \bar{z})=\bar{D} \tau_{20}^{*}\left(1, \bar{\alpha}^{*}, \bar{\beta}^{*}, \bar{\gamma}^{*}\right)\left(\begin{array}{l}
f_{1}(0) \\
f_{2}(0) \\
f_{3}(0) \\
f_{4}(0)
\end{array}\right) \\
= & \bar{D} \tau_{20}^{*}\left\{-\frac{r u_{1 t}^{2}(0)}{x_{M}}-\beta_{1} u_{1 t}(0) u_{3 t}(0)-\beta_{2} u_{1 t}(0) u_{2 t}(0)\right. \\
& +\bar{\alpha}^{*}\left[\beta_{1} e^{-m \tau_{1}} u_{1 t}\left(-\frac{\tau_{1}}{\tau_{20}^{*}}\right) u_{3 t}\left(-\frac{\tau_{1}}{\tau_{20}^{*}}\right)\right. \\
& \left.+\beta_{2} e^{-m \tau_{1}} u_{1 t}\left(-\frac{\tau_{1}}{\tau_{20}^{*}}\right) u_{2 t}\left(-\frac{\tau_{1}}{\tau_{20}^{*}}\right)\right] \\
& \left.-\bar{\beta}^{*} p u_{3 t}(0) u_{4 t} 0+\bar{\gamma}^{*} c u_{4 t}(-1) u_{3 t}(-1)\right\} .
\end{aligned}
$$

$$
\begin{aligned}
g_{20}= & 2 \bar{D} \tau_{20}^{*}\left[-\frac{r}{x_{M}}-\beta_{1} \beta-\beta_{2} \alpha+\overline{\alpha^{*}}\left(\beta_{1} e^{-m \tau_{1}} \beta e^{-2 i \omega_{20}^{*} \tau_{1}}+\beta_{2} e^{-m \tau_{1}} \alpha e^{-2 i \omega_{20}^{*} \tau_{1}}\right)-\overline{\beta^{*}} p \beta \gamma+\overline{\gamma^{*}} c \beta \gamma e^{-2 i \omega_{20}^{*} \tau_{20}^{*}},\right] \\
g_{11}= & \bar{D} \tau_{20}^{*}\left[-\frac{2 r}{x_{M}}-\beta_{1}(\beta+\bar{\beta})-\beta_{2}(\alpha+\bar{\alpha})+\overline{\alpha^{*}}\left(\beta_{1} e^{-m \tau_{1}}(\bar{\beta}+\beta)+\beta_{2} e^{-m \tau_{1}}(\bar{\alpha}+\alpha)\right)-\overline{\beta^{*}} p(\bar{\beta} \gamma+\beta \bar{\gamma})+\overline{\gamma^{*}} c(\bar{\beta} \gamma+\beta \bar{\gamma})\right], \\
g_{02}= & 2 \bar{D} \tau_{20}^{*}\left[-\frac{r}{x_{M}}-\beta_{1} \bar{\beta}-\beta_{2} \bar{\alpha}+\overline{\alpha^{*}}\left(\beta_{1} e^{-m \tau_{1}} \bar{\beta} e^{2 i \omega_{20}^{*} \tau_{20}^{*}}+\beta_{2} e^{-m \tau_{1}} \bar{\alpha} e^{2 i \omega_{20}^{*} \tau_{20}^{*}}\right)-\overline{\beta^{*}} p \bar{\beta} \bar{\gamma}+\overline{\gamma^{*}} c \bar{\beta} \bar{\gamma} e^{2 i \omega_{20}^{*} \tau_{20}^{*}}\right], \\
g_{21}= & 2 \bar{D} \tau_{20}^{*}\left\{-\frac{r}{x_{M}}\left[2 W_{11}^{(1)}(0)+W_{20}^{(1)}(0)\right]-\beta_{1}\left[\beta W_{11}^{(1)}(0)+\frac{1}{2} \bar{\beta} W_{20}^{(1)}(0)+W_{1}^{(3)}(0)+\frac{1}{2} W_{20}^{(3)}(0)\right]\right. \\
& -\beta_{2}\left[\alpha W_{11}^{(1)}(0)+\frac{1}{2} \bar{\alpha} W_{20}^{(1)}(0)+W_{1}^{(2)}(0)+\frac{1}{2} W_{20}^{(2)}(0)\right] \\
& +\overline{\alpha^{*}}\left[\beta_{1} e^{-m \tau_{1}}\left(\beta_{1} e^{-i \omega_{20}^{*} \tau_{20}^{*}} W_{11}^{(1)}\left(-\frac{\tau_{1}}{\tau_{20}^{*}}\right)+\frac{1}{2} \bar{\beta} e^{i \omega_{20}^{*} \tau_{20}^{*}} W_{20}^{(1)}\left(-\frac{\tau_{1}}{\tau_{20}^{*}}\right)+e^{-i \omega_{20}^{*} \tau_{20}^{*}} W_{11}^{(3)}\left(-\frac{\tau_{1}}{\tau_{20}^{*}}\right)+\frac{1}{2} e^{i \omega_{20}^{*} \tau_{20}^{*}} W_{20}^{(3)}\left(-\frac{\tau_{1}}{\tau_{20}^{*}}\right)\right)\right. \\
& \left.+\beta_{2} e^{-m \tau_{1}}\left(\alpha e^{-i \omega_{20}^{*} \tau_{20}^{*}} W_{11}^{(1)}\left(-\frac{\tau_{1}}{\tau_{20}^{*}}\right)+\frac{1}{2} \bar{\alpha} e^{i i \omega_{20}^{*} \tau_{20}^{*}} W_{20}^{(1)}\left(-\frac{\tau_{1}}{\tau_{20}^{*}}\right)+e^{-i \omega_{20}^{*} \tau_{20}^{*} W_{11}^{(2)}}\left(-\frac{\tau_{1}}{\tau_{20}^{*}}\right)+\frac{1}{2} e^{i \omega_{20}^{*} \tau_{20}^{*}} W_{20}^{(2)}\left(-\frac{\tau_{1}}{\tau_{20}^{*}}\right)\right)\right] \\
& -\overline{\beta^{*}} p\left[\beta W_{11}^{(4)}(0)+\frac{1}{2} \bar{\beta} W_{20}^{(4)}(0)+\gamma W_{11}^{(3)}(0)+\frac{1}{2} \bar{\gamma} W_{20}^{(3)}(0)\right] \\
& +\overline{\gamma^{*} c}\left[\beta e^{-i \omega_{20}^{*} \tau_{20}^{*}} W_{11}^{(4)}(-1)+\frac{1}{2} \bar{\beta} e^{i \omega_{20}^{*} \tau_{20}^{*}} W_{20}^{(4)}(-1)+\gamma e^{-i \omega_{20}^{*} \tau_{20}^{*}} W_{11}^{(3)}(-1)+\frac{1}{2} \bar{\gamma} e^{i \omega_{20}^{*} \tau_{20}^{*}} W_{20}^{(3)}(-1)\right],
\end{aligned}
$$

where 


$$
\begin{aligned}
& W_{20}(\theta)=\frac{i g_{20}}{\omega_{20} \tau_{20}^{*}} q(0) e^{i \omega_{20} \tau_{20}^{*} \theta}+\frac{i \bar{g}_{02}}{3 \omega_{20} \tau_{20}^{*}} \bar{q}(0) e^{-i \omega_{20} \tau_{20}^{*} \theta}+E_{1} e^{2 i \omega_{20} \tau_{20}^{*} \theta} \\
& W_{11}(\theta)=-\frac{i g_{11}}{\omega_{20} \tau_{20}^{*}} q(0) e^{i \omega_{20} \tau_{20}^{*} \theta}+\frac{i \bar{g}_{11}}{\omega_{20} \tau_{20}^{*}} \bar{q}(0) e^{-i \omega_{20} \tau_{20}^{*} \theta}+E_{2},
\end{aligned}
$$

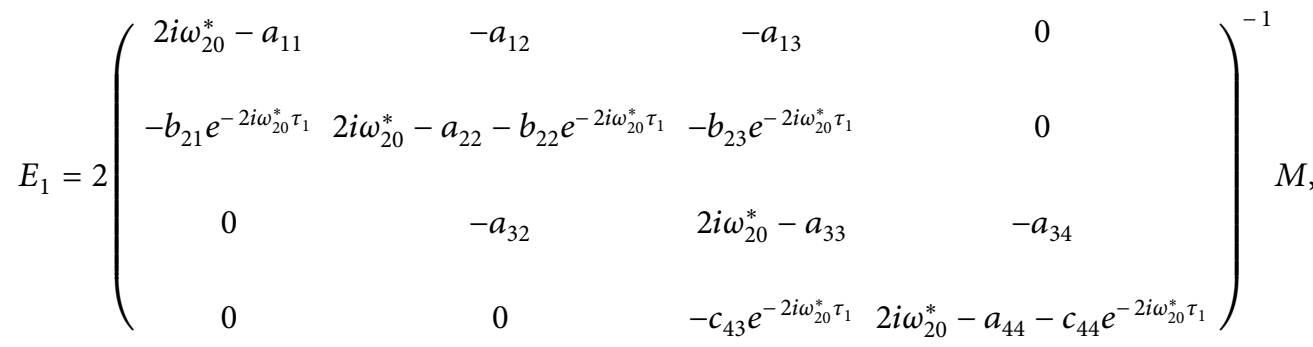

$$
\begin{aligned}
& E_{2}=\left(\begin{array}{cccc}
-a_{11} & -a_{12} & -a_{13} & 0 \\
-b_{21} & -a_{22}-b_{22} & -b_{23} & 0 \\
0 & -a_{32} & -a_{33} & -a_{34} \\
0 & 0 & -c_{43} & -a_{44}-c_{44}
\end{array}\right)^{-1} N \\
& M=\left(\begin{array}{c}
-\frac{r}{x_{M}}-\beta_{1} \beta-\beta_{2} \alpha \\
\beta \beta_{1} e^{-m \tau_{1}-2 i \omega_{20}^{*} \tau_{1}}+\alpha \beta_{2} e^{-m \tau_{1}-2 i \omega_{20}^{*} \tau_{1}} \\
-p \beta \gamma \\
c \beta \gamma e^{-2 i \omega_{20}^{*} \tau_{20}^{*}}
\end{array}\right), \\
& N=\left(\begin{array}{c}
-\frac{2 r}{x_{M}}-2 \beta_{1} \operatorname{Re}(\beta)-2 \beta_{2} \operatorname{Re}(\alpha) \\
2 \beta_{1} e^{-m \tau_{1}} \operatorname{Re}(\beta)+2 \beta_{2} e^{-m \tau_{1}} \operatorname{Re}(\alpha) \\
-2 p \operatorname{Re}(\bar{\beta} \gamma) \\
2 c \operatorname{Re}(\bar{\beta} \gamma)
\end{array}\right) .
\end{aligned}
$$

Next, we can calculate the following values: 

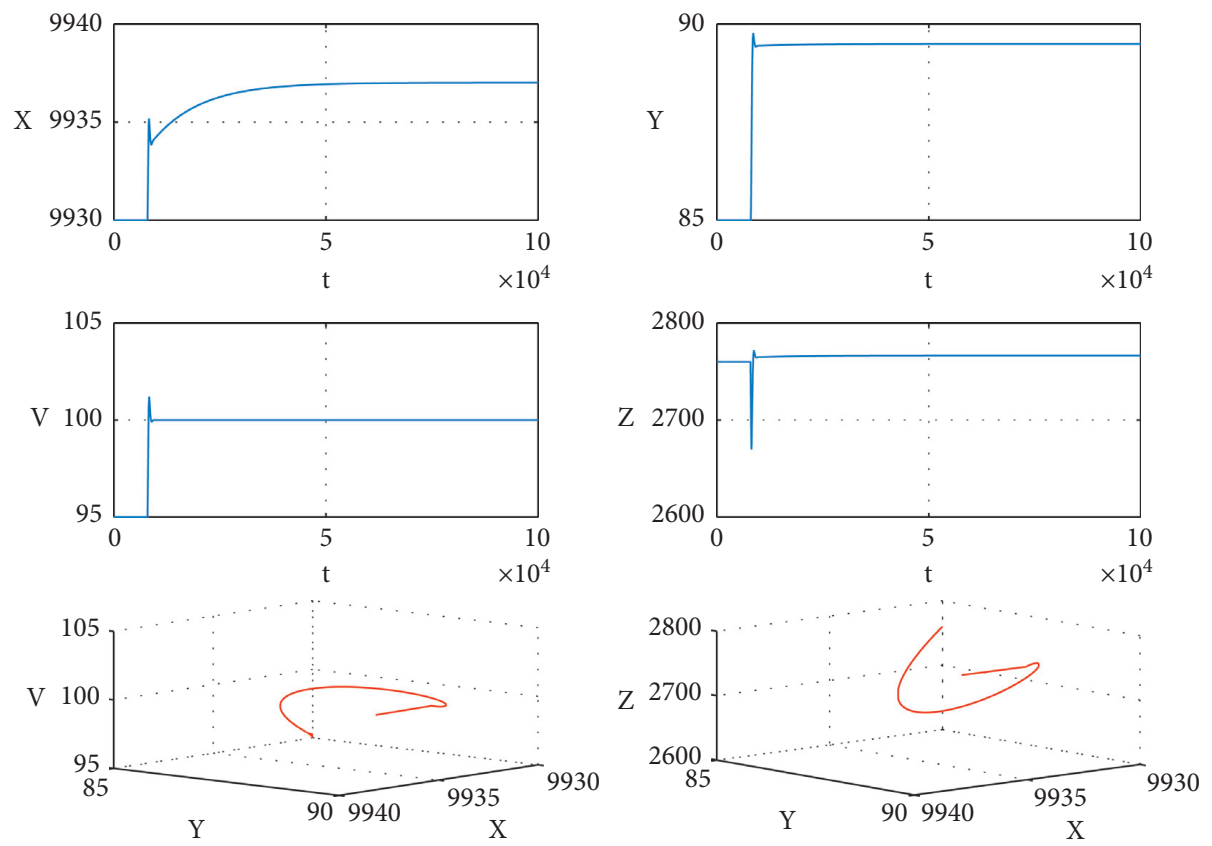

Figure 1: $E^{*}$ is asymptotically stable when $\tau_{1}=\tau_{2}=0$. The initial value is $9930,85,95$, and 2760 .

$$
\begin{aligned}
C_{1}(0) & =\frac{i}{2 \omega_{20}^{*} \tau_{20}^{*}}\left(g_{11} g_{20}-2\left|g_{11}\right|^{2}-\frac{\left|g_{02}\right|^{2}}{3}\right)+\frac{g_{21}}{2} \\
\mu_{2} & =-\frac{\operatorname{Re}\left(C_{1}(0)\right)}{\operatorname{Re}\left(\lambda^{\prime}\left(\tau_{0}^{*}\right)\right)} \\
T_{2} & =-\frac{\operatorname{Im}\left(C_{1}(0)\right)+\mu_{2} \operatorname{Im}\left(\lambda^{\prime}\left(\tau_{0}^{*}\right)\right)}{\omega_{20}^{*} \tau_{20}^{*}} \\
\beta_{2} & =2 \operatorname{Re}\left(C_{1}(0)\right)
\end{aligned}
$$

Then, for system (2), when $\tau_{1} \in\left[0, \tau_{1}^{*}\right), \tau_{2}>0, \tau_{1} \neq \tau_{2}$, the direction and the stability of periodic solution of Hopf bifurcation are determined by (59).

\section{Numerical Simulation}

In this section, we consider the following system:

$$
\left\{\begin{array}{l}
\frac{\mathrm{d} x(t)}{\mathrm{d} t}=r x(t)\left(1-\frac{x(t)}{10000}\right)-4.8 \times 10^{-5} x(t) v(t)-4.7 \times 10^{-5} x(t) y(t) \\
\frac{\mathrm{d} y(t)}{\mathrm{d} t}=4.8 \times 10^{-5} e^{-0.05 \tau_{1}} x\left(t-\tau_{1}\right) v\left(t-\tau_{1}\right)+4.7 \times 10^{-5} e^{-0.05 \tau_{1}} x\left(t-\tau_{1}\right) y\left(t-\tau_{1}\right)-a y(t) \\
\frac{\mathrm{d} v(t)}{\mathrm{d} t}=k y(t)-13 v(t)-0.06 v(t) z(t) \\
\frac{\mathrm{d} z(t)}{\mathrm{d} t}=0.01 v\left(t-\tau_{2}\right) z\left(t-\tau_{2}\right)-z(t)
\end{array}\right.
$$

and the numerical simulation is carried out under the following four cases:

(i) $\tau_{1}=\tau_{2}=0$.
Let $a=1, k=200$, and $r=0.01$, then we have $R_{1}=1.91>1$, and $\left(H_{1}\right)$ is satisfied. Meanwhile, $\Delta_{1}=179.53>0, \quad \Delta_{2}=2.97 \times 10^{4}>0, \quad \Delta_{3}=2.66 \times$ $10^{6}>0, \Delta_{4}=0.79>0$, and $\left(H_{2}\right)$ holds. Then, $E^{*}$ is 

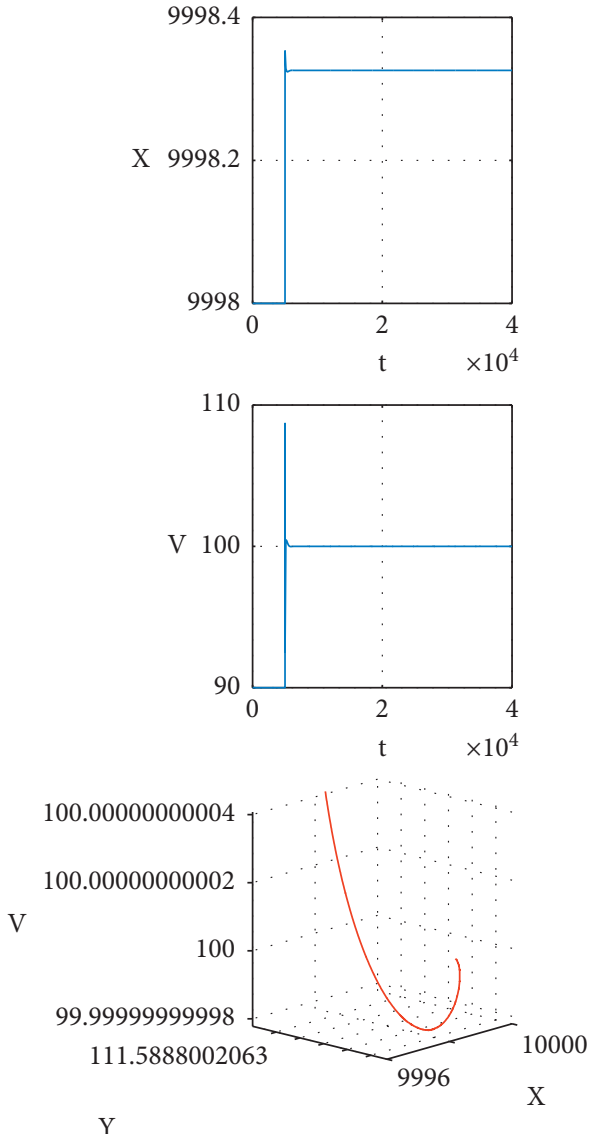
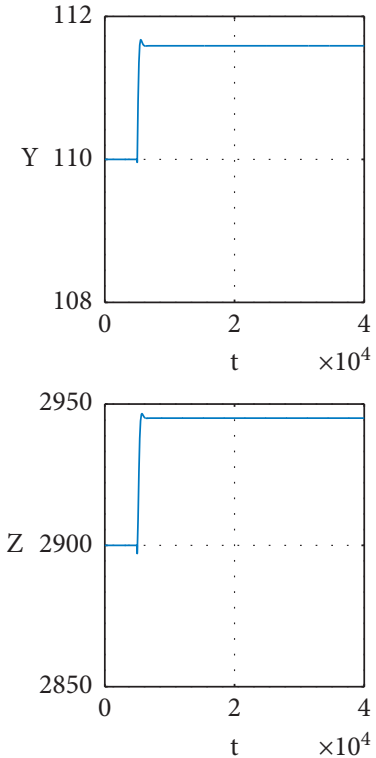

Figure 2: $E^{*}$ is asymptotically stable when $\tau_{1}=\tau_{2}=0$. The initial value is $9998,110,90$, and 2940 .
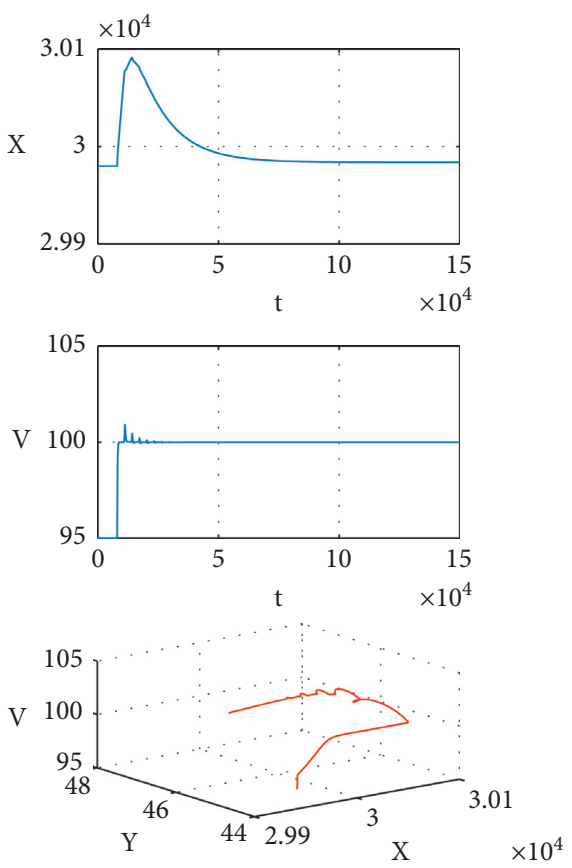
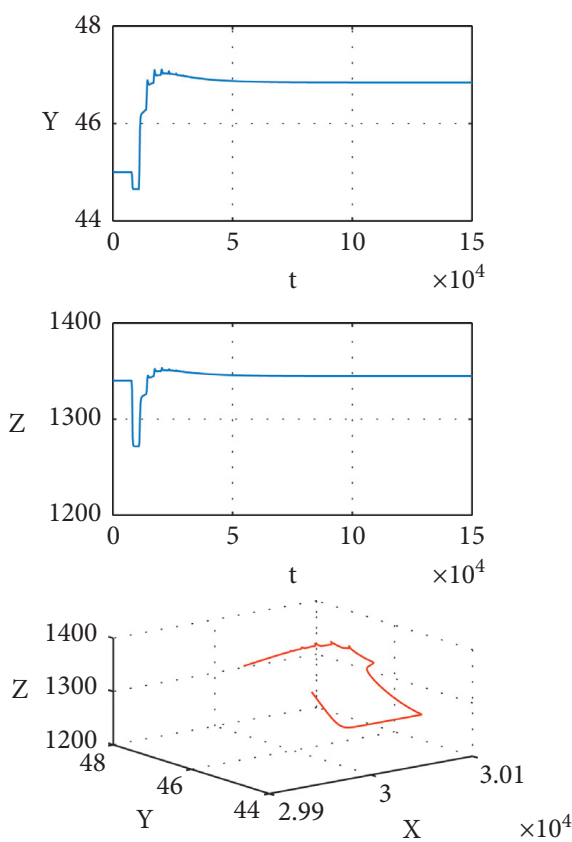

FIGURE 3: $E^{*}$ is asymptotically stable when $\tau_{1}=30$ and $\tau_{2}=0$. The initial value is $29980,45,95$, and 1340 . 

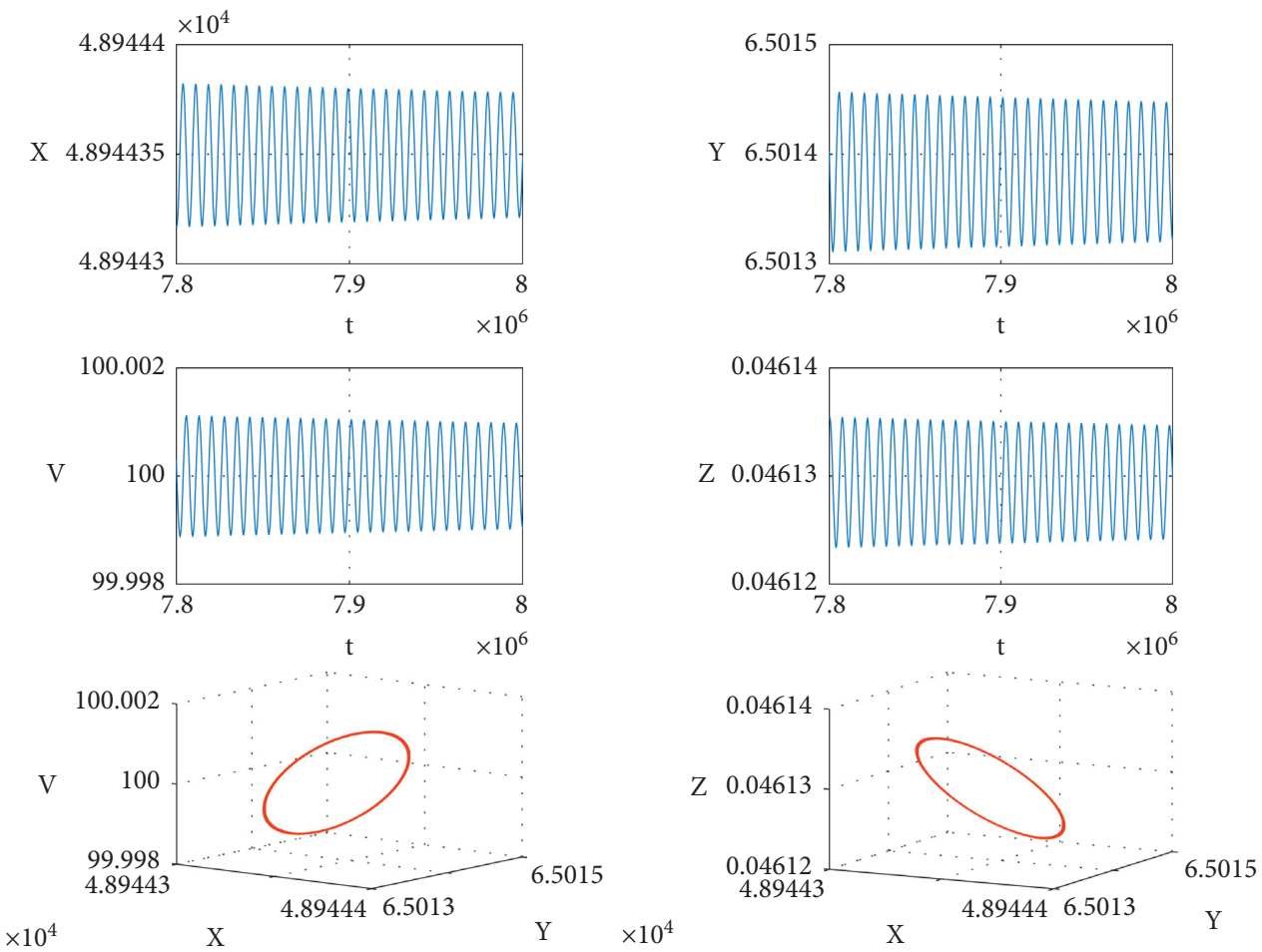

FIGURE 4: The trajectories of system (6) with $\tau_{1}=72.98$ and $\tau_{2}=0$. The initial value is $48930,6,95$, and 1 .
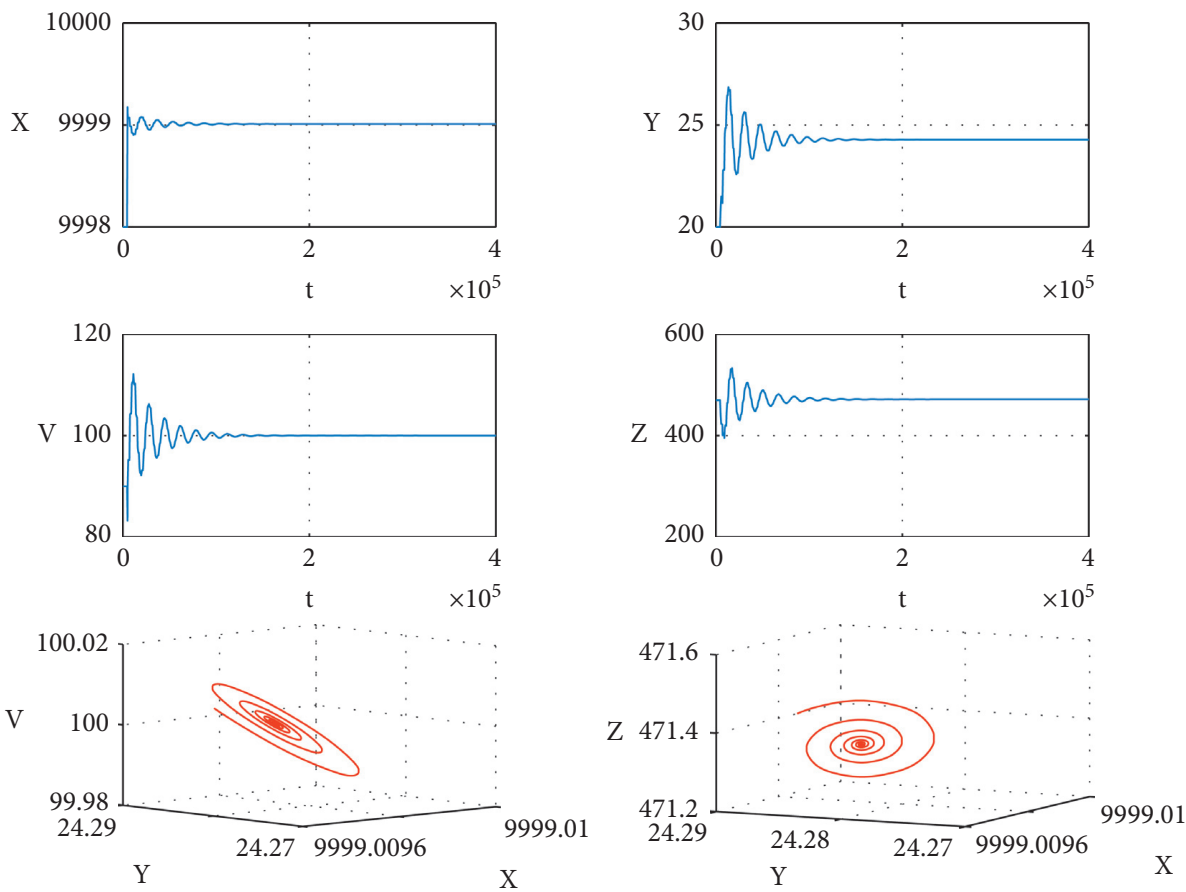

FIGURE 5: $E^{*}$ is asymptotically stable when $\tau_{1}=\tau_{2}=20$. The initial value is $9998,20,90$, and 470 . 

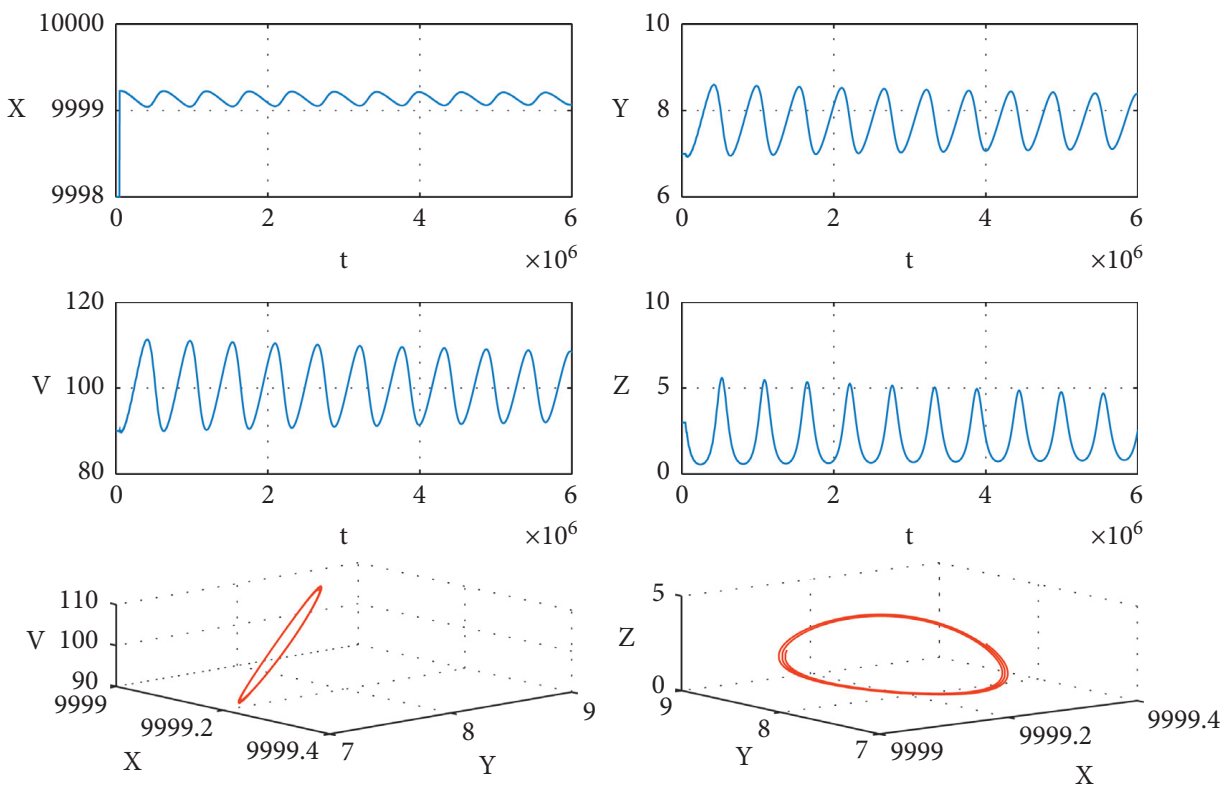

Figure 6: The trajectories of system (6) with $\tau_{1}=\tau_{2}=40.1$. The initial value is 9998, 7, 90, and 3 .
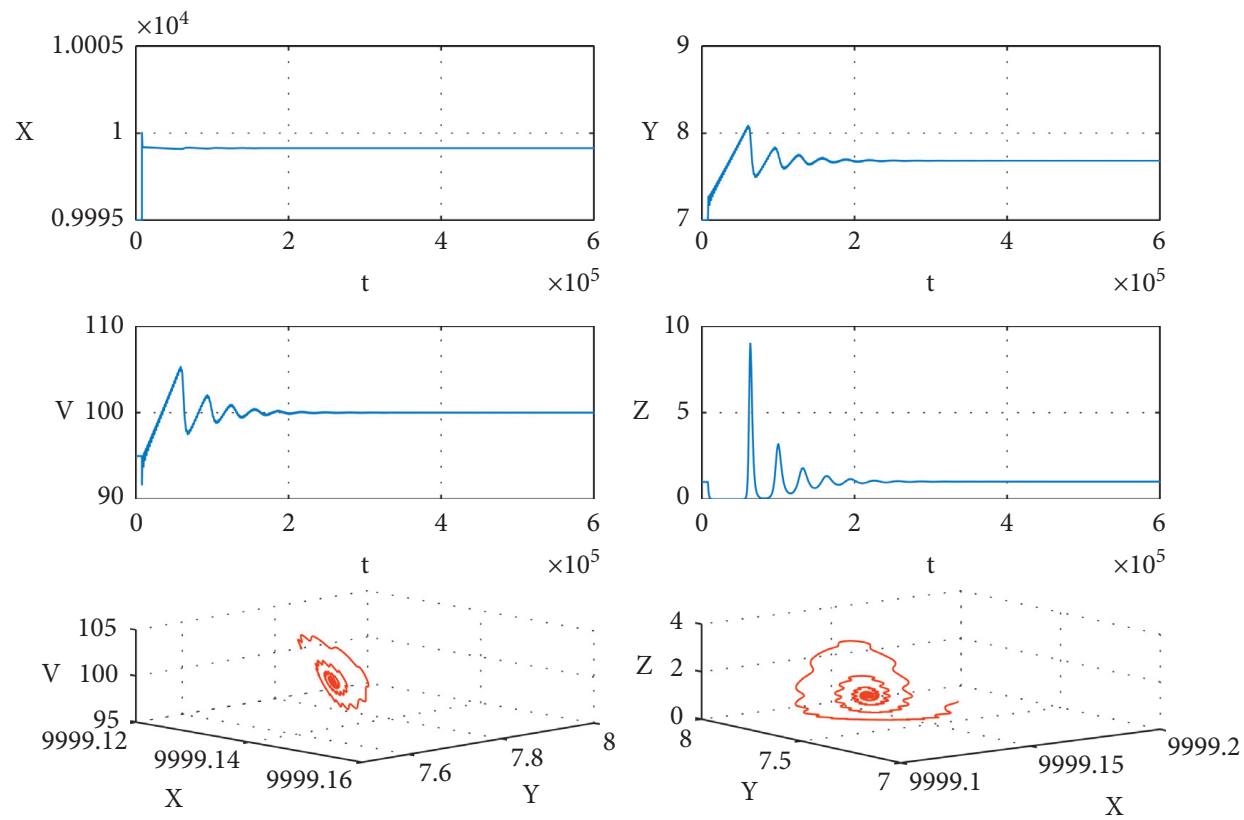

Figure 7: The trajectories of system (6) with $\tau_{1}=40.2$ and $\tau_{2}=0$. The initial value is 9995,7 , 95, and 1 .

locally asymptotically stable from Theorem 1 (see Figure 1).

Let $a=0.9, k=170$, and $r=60$; by simple calculation, we get $R_{1}=7.49>1$, and $\left(H_{1}\right)$ is satisfied. And $\quad \Delta_{1}=250.12>0, \quad \Delta_{2}=2.89 \times 10^{6}>0$, $\Delta_{3}=3.05 \times 10^{10}>0$, and $\Delta_{4}=4.56 \times 10^{3}>0$, we know that $\left(H_{2}\right)$ holds. Then, $E^{*}$ is locally asymptotically stable from Theorem 1 (see Figure 2).

(ii) $\tau_{1}>0$ and $\tau_{2}=0$.
Choosing $a=1, k=200$, and $r=0.01, E^{*}$ is locally asymptotically stable when $\tau_{1}=30$ (see Figure 3 ), and Hopf bifurcation occurs when $\tau_{1}=72.98$ (see Figure 4). It supports the results of Theorem 4 .

(iii) $\tau_{1}=\tau_{2}=\tau>0$.

Considering $a=0.9, k=170$, and $r=60$, we obtain $E^{*}$ is locally asymptotically stable when $\tau=20$ (see Figure 5), and Hopf bifurcation occurs when $\tau=40.1$ (see Figure 6). The results of Theorem 6 are verified. 

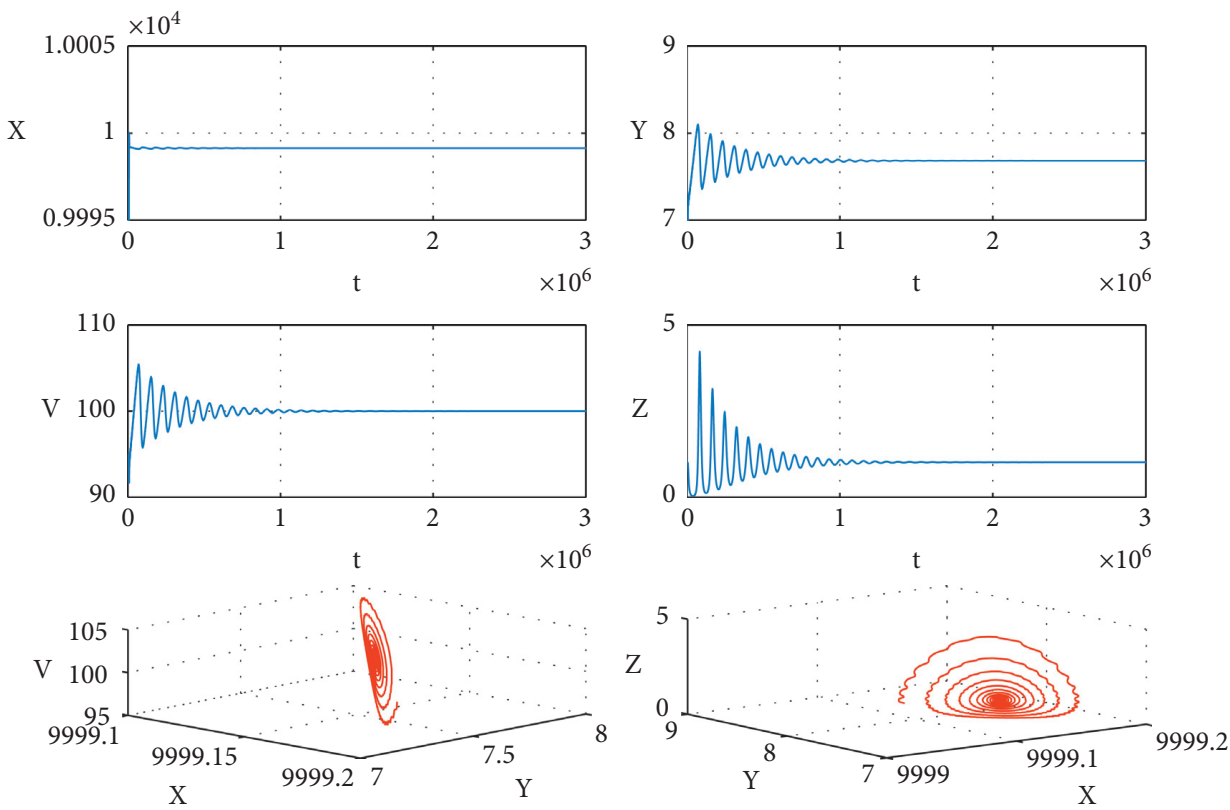

Figure 8: $E^{*}$ is asymptotically stable when $\tau_{1}=40.2$ and $\tau_{2}=5$. The initial value is $9995,7,95$, and 1 .
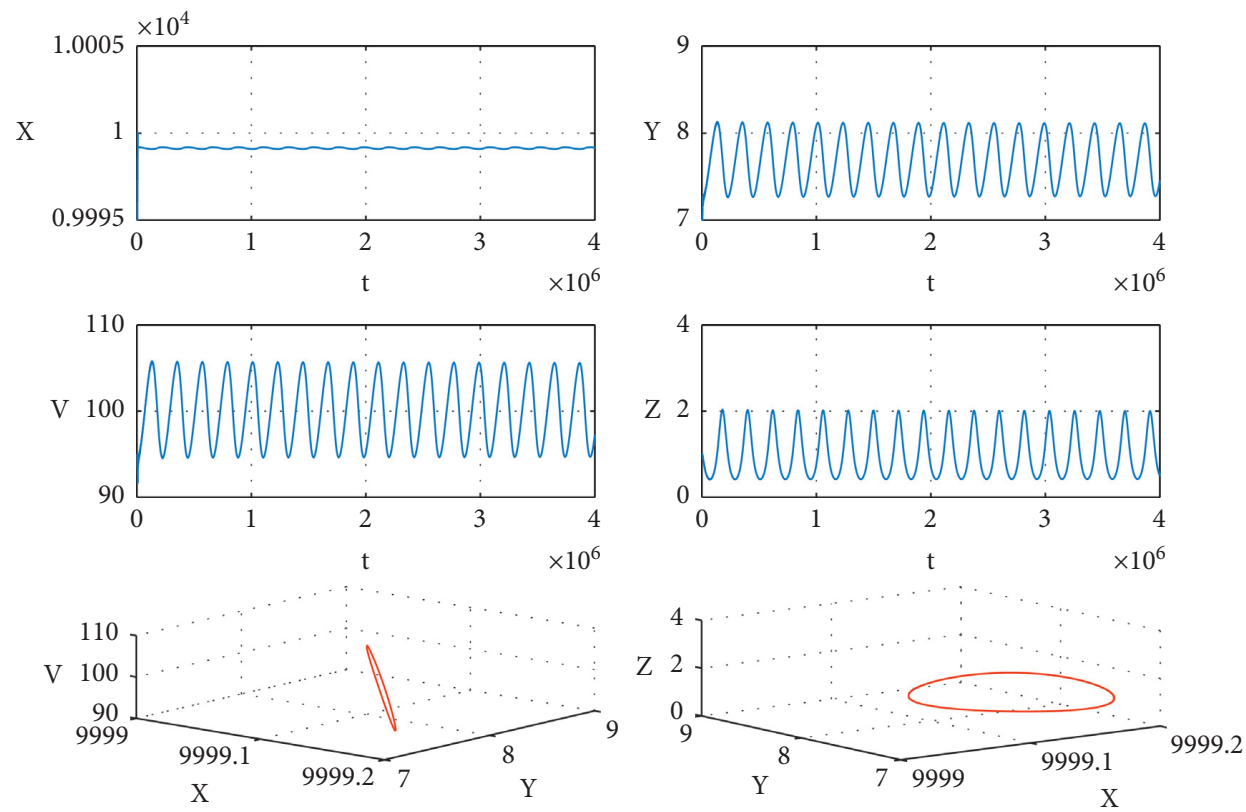

FIGURE 9: The trajectories of system (6) with $\tau_{1}=40.2$ and $\tau_{2}=48$. The initial value is $9995,7,95$, and 1 .

(iv) $\tau_{1} \in\left(0, \tau_{\max }\right)$ and $\tau_{2}>0$

Considering $a=0.9, k=170$, and $r=60$, by simple calculation, we get $\tau_{\max }=40.29$ and we choose $\tau_{2}$ as a parameter and let $\tau_{1}=40.2$. When $\tau_{2}=0$ (see Figure 7 ) and $\tau_{2}=5$ (see Figure 8 ), $E^{*}$ is locally asymptotically stable. And when $\tau_{2}=48$, Hopf bifurcation occurs (see Figure 9).

\section{Conclusion}

On the basis of [10], considering that $e^{-m \tau_{1}}$ is more practical that stands for the probability of surviving the time period from $t-\tau_{1}$ to $t$, this paper establishes and investigates an HIV-1 virus model with two delay and delay-dependent parameters to describe both virus-to-cell and cell-to-cell transmissions. We find out basic reproductive rate $R_{1}$, and 
system (2) has the unique positive equilibrium $E^{*}$ when $R_{1}>1$. Without time delays, $E^{*}$ is asymptotically stable (see Figures 1 and 2). In other words, system (6) does not have any excitable nature. When $\tau_{2}=0$, there exists a threshold limit beyond which the stability exchange takes place and system shows periodic orbits around $E^{*}$ (Figures 3 and 4 ). It shows that the number of cells and virus can fluctuate in time. Similarly, we find out that as $\tau_{1}$ and $\tau_{2}$ vary, $E^{*}$ loses its stability and Hopf bifurcations occur when $\tau_{1}=\tau_{2}=\tau$ and $\tau_{1} \in\left(0, \tau_{\max }\right), \tau_{2}>0$ (see Figures 5-9). Moreover, we obtain the direction and stability of the Hopf bifurcation when $\tau_{1} \in\left[0, \tau_{1}^{*}\right), \tau_{2}>0$ using the normal form theory and center manifold theorem. Finally, numerical simulations help us illustrate the main results of model (2). Clearly, we can show that delay value is responsible for changing the stable system to system with periodic cycles. In the discussion of the characteristic equations, when neither $\tau_{1}$ nor $\tau_{2}$ is zero, the characteristic equation of system (2) is $P_{0}\left(\lambda, \tau_{1}\right)+P_{1}(\lambda$, $\left.\tau_{1}\right) e^{-\lambda \tau_{1}}+P_{2}\left(\lambda, \tau_{1}\right) e^{-\lambda \tau_{2}}+P_{3}\left(\lambda, \tau_{1}\right) e^{-\lambda\left(\tau_{1}+\tau_{2}\right)}=0$. We only discuss about the case when $\tau_{1}=\tau_{2}=\tau>0$ by applying the geometric stability switch. When $\tau_{1} \neq \tau_{2}$, it is very difficult to discuss the root of such characteristic equation. We leave the analysis of the more complicated bifurcations as the future work.

\section{Data Availability}

The data used to support the findings of this study are included within the article.

\section{Conflicts of Interest}

The authors declare that there are no conflicts of interest in this paper.

\section{Acknowledgments}

This work was supported by the National Natural Science Foundation of China (No. 11761040).

\section{References}

[1] X. Lai and X. Zou, "Modeling cell-to-cell spread of HIV-1 with logistic target cell growth," Journal of Mathematical Analysis and Applications, vol. 426, no. 1, pp. 563-584, 2015.

[2] A. Murase, T. Sasaki, and T. Kajiwara, "Stability analysis of pathogen-immune interaction dynamics," Journal of Mathematical Biology, vol. 51, no. 3, pp. 247-267, 2005.

[3] J. Wang, J. Lang, and X. Zou, "Analysis of an age structured HIV infection model with virus-to-cell infection and cell-tocell transmission," Nonlinear Analysis: Real World Applications, vol. 34, pp. 75-96, 2017.

[4] S. Wang and D. Zou, "Global stability of in-host viral models with humoral immunity and intracellular delays," Applied Mathematical Modelling, vol. 36, pp. 1313-1322, 2002.

[5] J. Lin, R. Xu, and X. Tian, "Threshold dynamics of an HIV-1 virus model with both virus-to-cell and cell-to-cell transmissions, intracellular delay, and humoral immunity," Applied Mathematics and Computation, vol. 315, pp. 516-530, 2017.
[6] Y. Dong, G. Huang, R. Miyazaki, and Y. Takeuchi, "Dynamics in a tumor immune system with time delays," Applied Mathematics and Computation, vol. 252, pp. 99-113, 2015.

[7] F. Basir, S. Ray, and E. Venturino, "Role of media coverage and delay in controlling infectious diseases: a mathematical model," Applied Mathematics and Computation, vol. 252, pp. 99-113, 2015.

[8] Z. Zhang and H. Yang, "Stability and bifurcation in a stagestructured predator-prey system with holling-II functional response and multiple delays," International Journal of Computer Mathematics, vol. 92, no. 3, pp. 542-561, 2015.

[9] C. Xu and P. Li, "Dynamical analysis in a delayed predatorprey model with two delays," Discrete Dynamics in Nature and Society, vol. 2012, Article ID 652947, 22 pages, 2012.

[10] C. Sun, L. Li, and J. Jia, "Hopf bifurcation of an HIV-1 virus model with two delays and logistic growth," Mathematical Modelling of Natural Phenomena, vol. 337, pp. 372-385, 2018.

[11] X. Song, S. Wang, and J. Dong, "Stability properties and Hopf bifurcation of a delayed viral infection model with lytic immune response," Journal of Mathematical Analysis and Applications, vol. 373, no. 2, pp. 345-355, 2011.

[12] X. Shi, J. Cui, and X. Zhou, "Stability and Hopf bifurcation analysis of an eco-epidemic model with a stage structure," Nonlinear Analysis: Theory, Methods \& Applications, vol. 74, no. 4, pp. 1088-1106, 2011.

[13] J. Li and Z. Ma, "Stability switches in a class of characteristic equations with delay-dependent parameters," Nonlinear Analysis: Real World Applications, vol. 5, pp. 389-408, 2004.

[14] Q. An, E. Beretta, Y. Kuang, C. Wang, and H. Wang, "Geometric stability switch criteria in delay differential equations with two delays and delay dependent parameters," Journal of Differential Equations, vol. 266, pp. 7073-7100, 2018.

[15] Z. Jiang and Y. Guo, "Hopf bifurcation and stability crossing curve in a planktonic resource-consumer system with double delays," International Journal of Bifurcation and Chaos, vol. 30, no. 13, Article ID 2050190, 2020.

[16] E. Beretta and Y. Kuang, "Geometric stability switch criteria in delay differential systems with delay dependent parameters," SIAM Journal on Mathematical Analysis, vol. 33, no. 5, pp. 1144-1165, 2002.

[17] E. Beretta and Y. Tang, "Extension of a geometric stability switch criterion," Funkcialaj Ekvacioj, vol. 46, no. 3, pp. 337-361, 2003.

[18] S. Ruan and J. Wei, "On the zeros of a third degree exponential polynomial with applications to a delayed model for the control of testosterone secretion," Mathematical Medicine and Biology, vol. 18, no. 1, pp. 41-52, 2001. 